Karin Arnold, Carmen Dienst, Stefan Lechtenböhmer

\title{
Integrierte Treibhausgasbewertung der Prozessketten von Erdgas und industriellem Biomethan in Deutschland
}

Ursprünglich veröffentlicht als:

Karin Arnold, Carmen Dienst, Stefan Lechtenböhmer (2010):

Integrierte Treibhausgasbewertung der Prozessketten von Erdgas und industriellem Biomethan in Deutschland

In: Umweltwissenschaften und Schadstoff-Forschung, 22, 135-152

DOI: $10.1007 / \mathrm{s} 12302-010-0125-6$ 
Karin Arnold, Carmen Dienst ${ }^{*}$, Stefan Lechtenböhmer

\section{Integrierte Treibhausgasbewertung der Prozessketten von Erdgas und industriellem Biomethan in Deutschland}

Wuppertal Institut für Klima, Umwelt, Energie $\mathrm{GmbH}$, Deutschland

* Korrespondenz-Autorin: Carmen Dienst, Wuppertal Institut für Klima, Umwelt, Energie GmbH, Döppersberg 19, 42103 Wuppertal, Deutschland

E-mail: carmen.dienst@wupperinst.org 


\title{
Integrierte Treibhausgasbewertung der Prozessketten von Erdgas und industriellem Biomethan in Deutschland
}

\begin{abstract}
Zusammenfassung
Hintergrund. Die Bezugsquellen und Transportwege von fossilem Erdgas werden sich in den kommenden beiden Dekaden diversifizieren. Veränderungen der Lieferstruktur, verbunden mit weiteren Transportentfernungen und Neubau von Pipelines, sowie der verstärkte Einsatz von verflüssigtem Erdgas (LNG - Liquefied Natural Gas) sind zu erwarten. Entsprechend werden sich auch die vorgelagerten Prozessketten und die damit verknüpften THG-Emissionen verändern. Im Sinne einer korrekten und ganzheitlichen Bilanzierung der Lebenszyklusemissionen und weitgehender Treibhausgasminderungsziele, sind die vorgelagerten Emissionen eine nicht zu vernachlässigende Größe. Gleichzeitig wird Biomethan als Beimischung zum fossilen Erdgas an Bedeutung gewinnen. Obwohl seine Verbrennung als klimaneutral gewertet wird, sind die Prozesse zur Herstellung von Biomethan mit Emissionen verbunden.

Die Treibhausgas-Emissionen (THG) der Vorketten von in der EU eingesetzten Energieträgern werden in der neuen EUKraftstoffqualitätsrichtlinie reguliert (vom Dez. 2008). Ihre Höhe und Entwicklung wird für die klimapolitischen Diskussionen und politische Entscheidungen somit immer wichtiger.

Ziel. Vor dem Hintergrund der angesprochenen Aspekte sollen die zukünftige Entwicklung der Gasversorgung in Deutschland und die Veränderungen der vorgelagerten THG-Emissionen von Erdgas und Biomethan ermittelt werden. In zwei Szenarien werden die mit der Herstellung und dem Transport von Erdgas und Biomethan verknüpften Emissionen bis zum Jahr 2030 einschließlich des zu erwartenden technischen Optimierungspotenzials bilanziert. Mittels dieser Analyse können Einschätzungen der zukünftigen Emissionspfade und der durchschnittlichen Emissionen (Klimaqualität) des eingesetzten Gases (als Mischung fossiler und biogener Gase einschließlich der damit verbundenen Prozesskettenemissionen)
\end{abstract} gegeben werden. Diese können als Grundlage für energie- und klimapolitische Entscheidungen dienen.

Ergebnisse und Diskussion. Nach Erläuterung der Prozesskette von Biomethan werden die zu erwartenden technischen Entwicklungen der einzelnen Prozessschritte (Substratbereitstellung, Fermentierung, Aufbereitung, Gärrestnutzung) diskutiert und die Höhe der hiervon zu erwartenden Emissionen bilanziert. Basis sind Ergebnisse der wissenschaftlichen Begleitforschung des Wuppertal Instituts zur Einspeisung von Biomethan ins Erdgasnetz. Dabei gehen wir davon aus, dass die nächste Anlagengeneration ,Optimierte Technik' das aus heutiger Sicht bestehende Optimierungspotential des heutigen Stands der Technik ausschöpfen wird, so dass sich die spezifischen, auf den Heizwert des Biomethan bezogenen THGEmissionen der Vorkette von aktuell 27,8 t CO 2 -̈̈./TJ auf 14,8 t CO $2-\ddot{A} . / T J$ in 2030 fast halbieren werden.

Die zu erwartenden Emissionen der Erdgas-Prozesskette wurden in einem Vorgängerartikel bereits im Detail analysiert [vgl. Lechtenböhmer \& Dienst 2008]. Bei der Förderung und der Transportinfrastruktur ist ebenfalls eine Optimierung der Technik zu erwarten. Die dadurch erzielte Verringerung der spezifischen THG-Emissionen kann die aus den künftig längeren Transportstrecken und aufwendigen Produktionsprozessen resultierende Erhöhung ausgleichen.

Abschließend werden zwei Szenarien (Hoch- und Niedrigverbrauch) der künftigen Gasversorgung Deutschlands bis 2030 aufgestellt. Im Hochverbrauch-Szenario wird damit gerechnet, dass der Gaseinsatz in Deutschland um 17\% steigen wird. Im Niedrigverbrauchs-Szenario wird er dagegen um etwa $17 \%$ sinken. Gleichzeitig wird der Anteil von Biomethan am eingesetzten Gas auf 8 bzw. $12 \%$ ansteigen. Die - direkten und indirekten - Treibhausgasemissionen der Gasnutzung in Deutschland werden im Niedrigverbrauchs-Szenario um 25\%, d.h. überproportional von 215,4 Mio. t CO 2 - Äq auf 162,4 Mio. t $\mathrm{CO}_{2}$ - $\mathrm{Äq}$ zurückgehen. Im Hochverbrauchsszenario steigen die Gesamtemissionen leicht an, um 7\% (auf 230,9 $\mathrm{Mio}^{\mathrm{t}} \mathrm{CO}{ }_{2}$ Ä.).

Schlussfolgerungen. Gasförmige Energieträger werden in den kommenden beiden Dekaden eine zentrale Säule der deutschen Energieversorgung bleiben. Insgesamt zeigt sich, dass die THG-Emissionen der Nutzung von Erdgas v.a. von den Verbrauchsmengen der Gasversorgung abhängig sind. Das heißt, dass sowohl aus klima- als auch aus energiepolitischer Sicht die Steigerung der Energieeffizienz ein zentraler Faktor ist. Daneben bestehen sowohl in der verstärkten Nutzung von Biomethan als auch in der weiteren Investition in emissionsoptimierte Technologien entlang der Vorketten signifikante Emissionsminderungspotentiale. Hierdurch kann die ,Klimaqualität', d.h. die spezifische Treibhausgasemissionshöhe über alle Prozessstufen, des eingesetzten Gases deutlich verbessert werden. Die spezifischen Gesamtemissionen pro TJ eingesetzten Gases werden hierdurch um ca. 9\% von heute $63,3 \mathrm{t} \mathrm{CO}_{2}$-Äq. pro TJ auf etwa 54,5 t/TJ sinken. Entscheidend ist hierfür der verstärkte Einsatz von Biomethan, dessen Verbrennung aufgrund der biogenen Herkunft des Kohlenstoffs weitgehend klimaneutral ist (im Vergleich zu direkten Emissionen von $56 \mathrm{t} \mathrm{CO}_{2} / \mathrm{TJ}$ bei der Verbrennung von Erdgas oder 111 t $\mathrm{CO}_{2} /$ TJ bei z.B. Braunkohle). Die Vorteile der gasförmigen Energieträger in der Klimaqualität und effizienten Nutzung werden - insbesondere auch in der künftig zu erwartenden Beimischung von Biomethan - auch zukünftig Bestand haben.

Schlagwörter: Biomethan; Energiebereitstellung; Erdgas; Erdgastransport; EU-Kraftstoffqualitätsrichtlinie; LCA; Life cycle assessment; Prozessketten; THG (Treibhausgas)-Emissionen 


\begin{abstract}
Background. The use of natural gas has increased in the last years. In the future, its import supply and transport structure will diversify (longer distances; higher share of LNG (liquefied natural gas); new pipelines). Thus the process chain and GHG emissions of the production, processing, transport and distribution might change. Simultaneously, the injection of bio methane into the natural gas grid is becoming more important. Although its combustion is regarded as climate neutral, during the production processes of bio methane GHG emissions are caused.

The GHG emissions occurring during the process chain of energy fuels are relevant for the discussion on climate policy and decision making processes. They are becoming even more important, considering the new Fuel Quality Directive of the EU (Dec. 08), which aims at controlling emissions of the fuel process chains.

Aim. In the context of the aspects outlined above the aim is to determine the future development of gas supply for Germany and the resulting changes in GHG emissions of the whole process chain of natural gas and bio methane. With the help of two gas consumption scenarios and a LCA of bio methane the amount of future emissions and emission paths until 2030 can be assessed and used to guide decision processes in energy policy.

Results and Discussion. The process chain of bio methane and its future technical development are outlined and the related emissions calculated. The analysis is based on an accompanying research study on the injection of bio methane to the German gas grid. Two types of biogas plants have been considered whereof the "optimised technology" is assumed to dominate the future market. This is the one which widely exploits the potential of process optimisation of the current "state of the art" plant. The specific GHG emissions of the process chain can thus be nearly halved from currently $27.8 \mathrm{t} \mathrm{CO} \mathrm{C}_{2}$-eq./TJ to $14,8 \mathrm{t}$ $\mathrm{CO}_{2}$-eq./TJ in 2030 .

GHG emissions of the natural gas process chain have been analysed in detail in a previous article [see Lechtenböhmer \& Dienst 2008]. Significant modifications and a decrease of specific emissions is possible, depending on the level of investment in the modernisation of the gas infrastructure and the process improvements. These mitigation options might neutralise the emission increase resulting from longer distances and energy intensive processes.

In the last section two scenarios (low and high consumption) illustrate the possible development of the German gas supply until 2030, given an overall share of $8-12 \%$ of bio methane. Considering the dynamic emission factors calculated in the former sections, the overall gas emissions and average specific emissions of German gas supply can be given. The current emissions of 215.4 million $\mathrm{t} \mathrm{CO}_{2}$-eq. are reduced by $25 \%$ in the low-consumption scenario (162 million $\mathrm{t} \mathrm{CO}_{2}$-eq.), where consumption is reduced by $17 \%$. Assuming a consumption which is increased by $17 \%$ in 2030 , emissions are around $7 \%$ higher (230.9 million $\mathrm{t} \mathrm{CO}_{2}$-eq.) than today.

Conclusions. Gaseous fuels will still play a significant role for the German energy supply in the next two decades. The GHG emissions mainly depend on the amount of gas used. Thus, energy efficiency will be a key issue in the climate and energy related policy discussion. A higher share of bio methane and high investments in mitigation and best available technologies can significantly reduce the emissions of the process chain. The combustion of bio methane is climate neutral compared to 56 t $\mathrm{CO}_{2} / \mathrm{TJ}$ caused by the direct combustion of natural gas (or $111 \mathrm{t} \mathrm{CO}_{2} / \mathrm{TJ}$ emitted by lignite). The advantage of gaseous energy carriers with the lowest levels of GHG emissions compared to other fossil fuels still remains. This holds true for fossil natural gas alone as well as for the expected future blend with bio-methane.
\end{abstract}

Keywords: Bio-methane; Energy supply; Fuel Quality Directive; GHG-emissions; LCA; Life cycle assessment; Natural gas; Natural gas transport; Process chains

\title{
1 Hintergrund und Ausgangssituation
}

Erdgas hat als Energieträger in den letzten Jahrzehnten eine hohe Bedeutung im deutschen und europäischen Energiemarkt gewonnen und dabei in Deutschland einen Marktanteil von über 20\% am Primärenergieeinsatz erreicht. Dabei spielten auch die hohe Effizienz der Nutzung sowie die geringen direkten spezifischen Treibhausgasemissionen im Vergleich zu anderen fossilen Energieträger eine wichtige Rolle. Im Dezember 2008 hat die EU die neue Kraftstoffqualitätsrichtlinie [,Fuel Quality Directive'; European Parliament 2008] beschlossen und damit begonnen die Vorkettenemissionen der in den Ländern der EU eingesetzten Energieträger zu regulieren.

Mit Blick auf die Zukunft ergeben sich daraus mehrere Fragestellungen, die einen signifikanten Einfluss auf die Emissionsintensität der künftigen Gasversorgung haben.

(1) Die Versorgung mit fossilem Erdgas wird in den kommenden Jahren aus immer weiter entfernt liegenden sowie schwerer zu erschließenden Quellen und vermehrt als verflüssigtes Erdgas (LNG) erfolgen. Gleichzeitig wird es nach Einschätzung z.B. von Lechtenböhmer und Dienst [2008] zu einer zumeist altersbedingten Erneuerung der Infrastrukturen mit vielfach emissionsärmeren Technologien kommen ${ }^{1}$. Die potentiellen Auswirkungen dieser Trends auf die indirekten Emissionen des Gasimports nach Deutschland, die mit der künftigen Förderung, Aufbereitung und dem Transport des Erdgases verbunden sind, wurden in Lechtenböhmer \& Dienst [2008] bereits näher beleuchtet. Sie werden hier aufgegriffen und im Kontext der Diskussion künftiger Trends zur Klimaqualität des in Deutschland genutzten Gases neu bewertet.

1 Die Emissionen werden vor allem durch effizientere Turbinen sowie verbesserte, d.h. vor allem dichtere Leitungsnetze vermindert werden, wie dies dem heutigen Stand der Technik entspricht. Eine Übersicht über verschiedenste Möglichkeiten der Emissionsminderung an Erdgasfernleitungen geben z.B. Lechtenböhmer et al. (2007). 
(2) Die zweite relevante Entwicklung im Gasmarkt wird in einer verstärkten Nutzung und Beimischung von Biomethan zum fossilen Erdgas liegen. Diese soll in den kommenden Jahren deutlich ausgebaut werden und Biomethan wird bis 2030 einen relevanten Anteil an der Gasversorgung in Deutschland erreichen. Aufgrund seiner biogenen Herkunft sind die direkten $\mathrm{CO}_{2-}$ Emissionen der Verbrennung von Biomethan nicht klimarelevant. Biomethan wird regional produziert und direkt in das deutsche Gasnetz eingespeist. Dabei kommt es allerdings zu Treibhausgasemissionen aus der Vorkette der Biomethanbereitstellung, die in einer Gesamtbilanz nicht vernachlässigt werden dürfen. Ihre aktuelle und zukünftige Größenordnung wird hier auf der Basis aktueller Mess- und LCA-Ergebnisse näher analysiert.

Dieses Papier entwickelt vor diesen Hintergründen zwei Szenarien zu den mit der Gasversorgung in Deutschland verbundenen Treibhausgasemissionen. Diese bis 2030 reichenden Szenarien können u.a. als Grundlage für energie- und klimapolitische Entscheidungen genutzt werden. Hierfür werden in diesem Papier für beide Szenarien durchschnittliche Treibhausgasemissionsfaktoren unter Berücksichtigung der Vorkettenemissionen des in Deutschland genutzten Gases abgeleitet. Diese liefern wichtige Basisinformationen für die Umsetzung der Fuel Quality Directive [European Parliament 2008], die die Vorkettenemissionen von Energieträgern reguliert ${ }^{2}$.

Der erste Teil des Papiers widmet sich der über die kommenden zwei Jahrzehnte zu erwartenden technischen Entwicklung bei der Biomethangewinnung und den hiervon erwartenden THG-Emissionen. Er basiert auf den Ergebnissen des Wuppertal Instituts $^{3}$ aus der wissenschaftlichen Begleitforschung zur Einspeisung von Biomethan ins Erdgasnetz [Arnold \& Vetter 2010].

Der zweite Teil stellt Szenarien der künftigen Gasversorgung bis 2030 auf und diskutiert dabei die Einflussfaktoren auf entstehenden Treibhausgasemissionen über die gesamte Prozesskette, sowohl für das Biomethan als auch für das fossile Erdgas. Es ergeben sich daraus Szenarien für die künftige Entwicklung der Klimaqualität, d.h. der mittleren Treibhausgasemissionsfaktoren der Gasversorgung in Deutschland.

\section{Prozesskette: Biomethan zur Einspeisung}

Seit gegen Ende des Jahres 2006 die ersten beiden Anlagen zur Einspeisung von Biomethan ins Erdgasnetz nahezu zeitgleich in Betrieb genommen wurden hat insbesondere dieser spezielle Zweig der Bioenergie-Branche einen starken Aufschwung erlebt (Abbildung 1). In Pliening (Bayern) und Straelen (NRW) ist nicht nur die grundsätzliche Machbarkeit der Prozesskette ,Biogaserzeugung - Aufbereitung - Einspeisung ins Erdgasnetz' gezeigt worden, sondern gleichzeitig auch die Attraktivität der hierdurch erreichbaren Entkopplung von Energieerzeugung und Nutzung.

Abbildung 1: Biomethan zur Einspeisung in Deutschland, Stand: Februar 2009, kumuliert [verändert nach dena Einspeiseatlas, 2009]

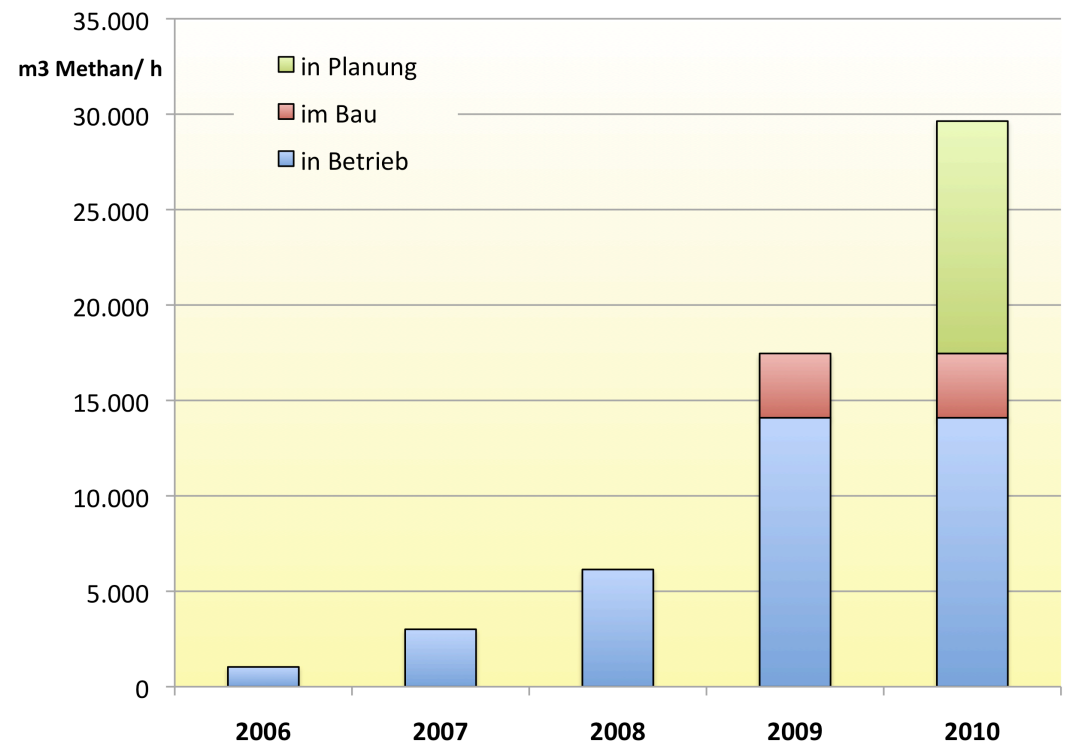

Musste Biogas bisher direkt an der Biogasanlage verstromt werden, so kann nach der Einspeisung ins Erdgasnetz das Gas über die bestehende Verteilinfrastruktur über nahezu beliebige Distanzen zum Endverbraucher transportiert werden. Der Vorteil besteht in energie- und klimapolitischer Sicht darin, dass der Ort der Verstromung so gewählt werden kann, dass die anfallende Abwärme ebenfalls genutzt werden kann. Dies ist bei der Nutzung vor Ort, also an einem landwirtschaftlichen

2 Sie können aber auch im Sinne einer ,Klimaqualität' von Energieträgern genutzt werden, über die ein neues klimapolitisches Instrumentarium zur Verringerung der Kohlenstoffintensität der genutzten Energieträger eingeführt werden könnte [vgl.Luhmann, Lechtenböhmer, Venjakob 2008].

3 Die hier vorgestellten Ergebnisse sind im Januar 2009 in einem Expertenworkshop unter Beteiligung des DBFZ, des Ifeu Instituts, der TLL sowie der E.ON Ruhrgas AG intensiv diskutiert worden. 
Betrieb in vielen Fällen nicht gegeben ${ }^{4}$. Als Erdgassubstitut kann Biomethan natürlich auch anderen Sektoren, wie der Wärmebereitstellung oder dem Kraftstoffmarkt zur Verfügung stehen.

Die Aufbereitung des erzeugten Biogases muss so erfolgen, dass die Beschaffenheit des im Erdgasnetz befindlichen Gases erreicht wird, damit der Endverbraucher keine Qualitätsschwankungen hinnehmen muss, die zudem die Abrechnung erschweren würden. Nach der erfolgten Aufbereitung spricht man dann von Biomethan.

Auf die technischen Anforderungen wird im Folgenden noch näher eingegangen; es sei aber vorausgeschickt, dass eine Aufbereitung sich erst ab einer bestimmten Größenordnung der Biogasanlage wirtschaftlich betreiben lässt [UMSICHT, 2008]. Vor dem Hintergrund der neuen Option, das erzeugte Gas über das Erdgasnetz zu vertreiben, ist die Größe der Biogasanlagen in Deutschland seit dem Jahr 2004 (Erteilung des Nawaro-Bonus im EEG) sichtbar angestiegen [Fachverband Biogas, 2007].

Gleichfalls ist $\mathrm{zu}$ bemerken, dass sich in den letzten zwei Jahren verstärkt auch größere Akteure wie z.B. Gasversorgungsunternehmen, im Sektor Biogas engagieren, wo vor dieser Zeit hauptsächlich Landwirte aktiv waren. Es findet eine Industrialisierung der Biogaserzeugung statt, mit der auch eine Professionalisierung im Betrieb der Anlagen einhergeht $^{5}$ [EnviTec, 2007]. Diese schlägt sich in der Effektivität und damit auch in der Treibhausgasbilanz der Prozessketten nieder. Die Klimaintensität und Ökobilanz von Biogas und Biomethan ist bereits in mehreren Studien untersucht worden [siehe etwa ifeu, 2008; KTBL, 2007; FNR, 2008; Wuppertal Institute et al. 2006]. [Soukup, 2008] hat gezeigt, dass ein Vergleich der Ergebnisse nicht immer möglich ist, da sie stark von der Substratwahl und Technik, aber vor allem von den getroffenen Annahmen sowie den Systemgrenzen abhängig sind. Zudem spielt die Wahl der funktionellen Einheit eine Rolle. Aus diesem Grund werden die relevanten Annahmen und Daten der vorgestellten Rechnungen in dem vorliegenden Artikel dargestellt.

An dieser Stelle sei ausdrücklich darauf hingewiesen, dass sich die hier abgebildete Treibhausgasbilanz ausschließlich auf großmaßstäblich und industriell geführte Biogasanlagen bezieht. Bei vielen der älteren und kleineren landwirtschaftlich betriebenen Anlagen kann es an verschiedenen Stellen (z.B. im Reaktor oder im Gärrestlager) zu teils erheblichen Leckagen von Methan kommen. Diese können im Extremfall dazu führen, dass der Klimaschutz-Vorteil der Prozesskette Biomethan gegenüber fossilem Erdgas ins Gegenteil verkehrt wird.

Solche Anlagen werden im Folgenden aber nicht untersucht, da der Fokus der Artikels auf industriellen Biogasanlagen zur Einspeisung liegt. Dabei ist $\mathrm{zu}$ beachten, dass die hier betrachteten Anlagen derzeit nicht den gesamten Bestand an Biogasanlagen in Deutschland abbilden.

\subsection{Technische Entwicklungslinien der Biomethanproduktion}

Als Grundlage für die Treibhausgasbilanz der Erzeugung von Biomethan wird im folgenden Absatz die Prozesskette ,Biomethan zur Einspeisung ins Erdgasnetz (frei Einspeisestelle)' skizziert. Die Prozesskette der Gewinnung von Biogas durch anaerobe Vergärung kann vereinfacht in die Abschnitte (1) Substratbereitstellung, (2) Fermentierung, (3) Aufbereitung unterteilt werden. Für die Bilanz der Prozesskette ist außerdem die (4) Nutzung der Gärreste relevant. Die Prozessschritte werden im Folgenden kurz beschrieben (Tabelle 1).

\subsubsection{Substratbereitstellung}

Als Substrate für die Vergärung kommen grundsätzlich unterschiedliche Pflanzen wie Mais, Getreide, Grassilage etc. in Frage, aber auch tierische Exkremente und biologische Abfälle. Die neue Anlagengeneration nutzt derzeit aber vorrangig solche Substrate, die gezielt für diesen Zweck angebaut worden sind - auf diese Art kann die Versorgung mit ausreichend Inputmaterial am besten sichergestellt werden, da Reststoffe in ihrem Mengenpotenzial begrenzt sind. Die Vergärung von Gülle und anderen Reststoffen spielt daher an dieser Stelle keine Rolle.

Bei der Auswahl der Ackerkulturen, die sich als Energiepflanze für die Biogasgewinnung eignen, sind der Hektarertrag und der Energieinhalt des Substrats, entscheidend, der sich im Methananteil des produzierten Gases widerspiegelt. Im Rahmen dieses Artikels wird vereinfacht nur Mais als gängigstes Substrat betrachtet. Die Pflanze ist sowohl hinsichtlich der Gasausbeuten als auch der Kosten im Anbau bislang die wirtschaftlichste Option. Wie in der Landwirtschaft üblich, kann der Mais in eine mehrgliedrige Fruchtfolge eingebunden werden, so dass den Anforderungen z.B. hinsichtlich einer aufgelockerten, möglichst heterogenen Landwirtschaft Genüge getan werden kann. [Arnold\&Vetter, 2010] zeigen, dass nach guter fachlicher Praxis in regional angepassten Fruchtfolgesystemen Biogassubstrate bereitgestellt werden können, die zu durchaus zufriedenstellenden THG-Bilanzen führen und gleichzeitig den Anforderungen einer nachhaltigen Landwirtschaft genügen.

Das Substrat wird geerntet, in unmittelbarer Nähe der Biogasanlage im Silo eingelagert und mit Folie abgedeckt. Die Bevorratung großer Mengen Biomasse ist erforderlich, denn die Erträge aus einer oder maximal zwei Ernten pro Jahr (abhängig von den eingesetzten Substraten) müssen für den dauerhaften Volllastbetrieb der Biogasanlage bis zur nächsten Ernteperiode zur Verfügung stehen. Die abgedeckte Lagerung dient dabei als biochemische Konservierungsmethode [Soukup, 2008]. 
Tabelle 1: Eingangsparameter der beiden Anlagentypen für die Ökobilanz (eigene Darstellung)

\begin{tabular}{|c|c|c|c|c|}
\hline & & & $\begin{array}{c}\text { Stand } \\
\text { der Technik }\end{array}$ & $\begin{array}{l}\text { Optimierte } \\
\text { Technik }\end{array}$ \\
\hline \multirow[t]{4}{*}{ Substratbereitstellung* } & Dieseleinsatz & I/ha & 82,9 & 82,9 \\
\hline & $\mathrm{N}$-Dünger & $\mathrm{kg} / \mathrm{ha}$ & 141,75 & 141,75 \\
\hline & Silierungsverluste im Vorlager & Massen \% & 15 & 5 \\
\hline & $\mathrm{N}_{2} \mathrm{O}$ Emissionen Boden & $\%^{* \star}$ & 1 & 1 \\
\hline \multirow[t]{2}{*}{ Fermenter } & Gasverlust BGA & Vol \% & 1 & 0,5 \\
\hline & Rohgasertrag & $\mathrm{m}^{3} / \mathrm{t} \mathrm{FM}$ & 200 & 220 \\
\hline \multirow[t]{4}{*}{ Gärrestmanagement } & Gasverlust Lager & $\%$ & 0 & 0 \\
\hline & $\mathrm{NH}_{4}-\mathrm{N}$ Anteil & $\%$ & 60 & 80 \\
\hline & $\begin{array}{l}\text { Ersatz von N-Dünger } \\
\text { (mineralisch) }\end{array}$ & $\%$ & 70 & 70 \\
\hline & $\begin{array}{c}\text { Ersatz von K,P-Dünger } \\
\text { (mineralisch) }\end{array}$ & $\%$ & 100 & 100 \\
\hline \multirow[t]{2}{*}{ Aufbereitung } & Methanverlust & $\%$ & 2 & 2 \\
\hline & Restemission & $\%$ & 0,01 & 0,01 \\
\hline \multirow[t]{4}{*}{ Energiebedarf } & Strombedarf BGA & $\mathrm{kWh}$ el/ t FM & 36 & 36 \\
\hline & Wärmebedarf BGA & $\mathrm{kWh}$ th/ t FM & 83 & 83 \\
\hline & Strombedarf PSA & $\mathrm{kWh} \mathrm{el} / \mathrm{m}^{3} \mathrm{BG}$ & 0,3 & 0,3 \\
\hline & Wärmebedarf PSA & $\mathrm{kWh}$ th $/ \mathrm{m}^{3} \mathrm{BG}$ & 0 & 0 \\
\hline
\end{tabular}

*exemplarisch für Mais, ohne Gärrestrückführung

** berechnet in \% des ausgebrachten Stickstoff-Düngers

\subsubsection{Fermentierung}

Aus dem Silo wird das Substrat dem Fermenter und dem eigentlichen Prozess der Gasbildung zugeführt. Bei Einsatz des weit verbreiteten kontinuierlichen oder quasi-kontinuierlichen Verfahrens ${ }^{6}$ wird hierfür durch einen Rad- oder Teleskoplader zunächst ein Dosierer beschickt, der mit Förderschnecken automatisch für einen gleichmäßigen Eintrag der Silage in den Fermenter sorgt. Eine entsprechend große Menge an vergorenem Substrat verlässt gleichzeitig den Reaktor. Es entsteht ein gleichmäßiger Durchfluss, dessen Geschwindigkeit die durchschnittliche Verweildauer der Biomasse im Fermenter bestimmt. Beides ist von der Geschwindigkeit der Umsetzung des Substrats zu Biogas abhängig [Soukup, 2008].

Unabhängig von der technischen Ausführung verläuft die Biogasgewinnung immer unter Licht- und Sauerstoffabschluss nach dem gleichen Prinzip der anaeroben Fermentation in den vier Stufen der Hydrolyse (Zerlegung des Ausgangsmaterials in einfache organische Bausteine), Acidogenese (Bildung von niederen Fettsäuren), Actegonese (Umwandlung der Zwischenprodukte zu hauptsächlich Essigsäure, Kohlenstoffdioxid und Wasserstoff) und Methanogenese (Methanbildung) [FNR, 2006].

\subsubsection{Aufbereitung}

Das so entstandene Produktgas besteht hauptsächlich aus Kohlenstoffdioxid und Methan. Die Stoffkonzentrationen der einzelnen Bestandteile des Biogases können insbesondere in Abhängigkeit von den Nährstoffzusammensetzungen der Ausgangssubstrate und der Betriebsführung der Anlage stark variieren. Um eine Einspeisung ins Erdgasnetz realisieren zu können, müssen die technischen Eigenschaften des Rohbiogases an die des Erdgases angepasst werden. Das bedeutet, es muss entschwefelt werden und das Kohlenstoffdioxid muss fast vollständig aus dem Gas entfernt werden. Hierdurch wird ein Methangehalt von bis zu $96 \%$ und mit annähernd $10 \mathrm{kWh} / \mathrm{m}^{3}$ der Heizwert von Erdgas H erreicht [FNR, 2008].

Die hierfür verfügbaren Verfahren - exemplarisch zu nennen sind Druckwasserwäsche (DWW) und Druckwechseladsorption (Pressure Swing Adsorption, PSA) auf der einen Seite, und die Aminwäsche als chemisches Verfahren auf der anderen Seite - unterscheiden sich im Hinblick auf den Eigenbedarf an elektrischer und thermischer Energie, die Methanverluste und ihre Betriebskosten [UMSICHT, 2008].

Im Anschluss an die biogasspezifische Aufbereitung sind weitere Konditionierungsschritte erforderlich: Der Druck des Gases muss an der Einspeisestelle oberhalb des Leitungsdrucks liegen, was in einer Gasdruckregel- und Messanlage sichergestellt

6 Daneben werden auch diskontinuierliche Verfahren eingesetzt, d.h. die vollständige Befüllung eines Fermenters erfolgt in einem Arbeitsgang. Zudem existieren eine Vielzahl unterschiedlicher Fermenterbauformen: stehende oder liegende Behälter aus Beton oder Stahl in verschiedenen Größen, oft ausgerüstet mit unterschiedlichsten Rührwerken zur Substratdurchmischung. In Abhängigkeit vom Trockenmassegehalt des Fermentationsraumes wird zwischen Nass- und Trockenfermentation unterschieden. 
wird. Eingespeist wird üblicherweise in ein Netz mittlerer Druckstufe, wie im Fall der hier abgebildeten Anlage bei 16 bar ${ }^{7}$. In einer zusätzlichen Gasbeschaffenheitsmessung werden die brenntechnischen Kenndaten des Gases überprüft und können bei Bedarf noch durch Zugabe von Konditionierungsgas präzise eingestellt werden. Schließlich erfolgt durch Beimischung von Geruchsstoffen eine aus Sicherheitsgründen vorgeschriebene Odorierung des bis dahin geruchlosen Gases [Wuppertal Institut et al., 2006].

\subsubsection{Gärrestnutzung}

Nach einer gewissen Verweildauer im Fermenter wird das dann weitgehend ausgefaulte Ausgangssubstrat, das jetzt als Gärrest bezeichnet wird, in einen Nachgärbehälter und schließlich in ein Gärrestlager überführt. Auch hier findet nach dem oben beschriebenen Prinzip noch Neubildung von Biogas statt, wenn auch in deutlich geringerem Ausmaß. Moderne Biogasanlagen verfügen über ein abgedecktes Gärrestlager. Unkontrolliertes Entweichen methanhaltiger und damit stark klimawirksamer Gase in die Atmosphäre wird so verhindert.

Die Mineralstoffe, die von den Energiepflanzen während ihres Wachstums aufgenommen wurden, bleiben nach der Fermentation zu einem Großteil im Gärrest zurück, so dass bei Rückführung dieses Gärrests von einem annährend geschlossenen Nährstoffkreislauf ausgegangen werden kann. Als Wirtschaftsdüngeeichr ersetzt das ausgefaulte Substrat hier zumindest teilweise den Zukauf von großtechnisch hergestellten Mineraldüngern: nach [Gutser, 2008] kann davon ausgegangen werden, dass der ausgebrachte Gärrest einen Düngewert von 50 - 70 \% gegenüber dem mineralischen Stickstoffdünger beinhaltet. Damit senkt die Gärrestnutzung die Kosten des Substratanbaus und reduziert die Umweltbelastung aus den Vorketten der Düngemittelproduktion. Eine entsprechende Gutschrift wird in die Bilanzierung der Treibhausgasemissionen mit eingeschlossen.

Die Möglichkeit, den Gärrest zu nutzen und so einen annährend geschlossenen Nährstoffkreislauf zu fahren, hebt die Biogas/ Biomethanproduktion positiv von anderen Biomassenutzungs-Optionen wie z. B. den Biokraftstoffen der ersten Generation ab, wo dies problematisch ist.

\subsection{Bilanz der Treibhausgasemissionen}

Für die Ermittlung der Treibhausgasemissionen der Biomethanaufbereitung wird zwischen zwei Anlagentypen unterschieden: (1) einer großtechnisch geführten Anlage nach heutigem ,Stand der Technik', die eine durchschnittlich effektive Biogasanlage im Jahr 2008 im industriellen Maßstab abbildet und (2) einer Anlage, die als ,Optimierte Technik' das aus heutiger Sicht und für die nahe Zukunft denkbare Optimierungspotenzial so weit wie möglich ausschöpft. Die Anlage nach dem Stand der Technik ist durch die Mitglieder des Expertenworkshop des Wuppertal Instituts als repräsentativ für die derzeitige Generation anerkannt worden; bei der letztgenannten Anlage (,optimierte Technik') handelt es sich um einen Anlagentyp, der bereits stellvertretend für die nächste Generation an Biogasanlagen steht. Hier sind im Wesentlichen Daten eines Anlagenbetreibers verwendet worden, die als ,obere (beste) Bandbreite' zu verstehen sind. Als Rohstoff wird exemplarisch von Mais ausgegangen (s.o.).

\subsubsection{Ableitung der Emissionsfaktoren für die Biomethan-Prozesskette}

Bei allen Schritten der o.g. Prozesskette können Treibhausgasemissionen entstehen: entweder indirekt durch den Verbrauch an Strom und Wärme, oder direkt durch Entweichen von Gas. Am stärksten schlagen direkte Leckagen von Methan zu Buche, da das Gas eine vielfach höhere Klimawirksamkeit als Kohledioxid aufweist. Spezifisch noch klimawirksamer sind allerdings die Ausgasungen von Lachgas (N2O), die im Zuge des Substrat- und Gärresthandlings auftreten. Außerdem muss für die Gesamtbetrachtung das Nebenprodukt Gärreststoffe berücksichtigt werden. Durch ihre Nutzung anstelle von Mineraldüngern wird sowohl die ökonomische als auch die Emissionsbilanz verbessert.

Im Folgenden werden die emissionsrelevanten Parameter auf den relevanten Prozessschritten näher diskutiert und Emissionsfaktoren für die beiden hier betrachteten Anlagentypen ,Stand der Technik' und ,Optimierte Technik' abgeleitet. Zur besseren Orientierung sind die Parameter in Tabelle 1 fett gedruckt, bei denen sich die beiden Anlagentypen bzw. Anlagenkonfigurationen unterscheiden.

\section{Substratbereitstellung:}

Da an dieser Stelle die Darstellung der Anlagentechnik im Vordergrund steht, wird keine Differenzierung nach unterschiedlichen Substraten oder hinsichtlich der beiden Anlagentypen vorgenommen. Exemplarisch wird die Bereitstellung von Mais als Biogassubstrat gesetzt. Die Substratbereitstellung beinhaltet den Anbau und die Ernte der Substrate sowie die Silierung im Vorlager.

Entscheidend für die THG Emissionen, die der Anbau des Substrats mit sich bringt, sind - neben dem Einsatz von Diesel in Arbeitsgeräten - die Aufwendungen für Stickstoff-, Phosphor- und Kaliumdünger, sowie für Magnesium, Kalk, Pflanzenschutzmittel und Saatgut. In Tabelle 1 sind von diesen nur der Dieseleinsatz und Stickstoff-Dünger als wichtigste Parameter aufgeführt, die den größten Anteil an den THG Emissionen bilden. Diese Werte sind im Rahmen der Arbeiten des Wuppertal Instituts von der Thüringischen Landesanstalt für Landwirtschaft (TLL) bestimmt worden und bilden die Situation an einem definierten Ackerstandort in Deutschland ab [Arnold\&Vetter, 2010]. Zudem sind Ausgasungen von Lachgas in der Höhe von $1 \%$ des ausgebrachten Stickstoffdüngers als THG Emissionen in die Bilanz einbezogen worden [IPCC, 2007].

7 http://www.biogaspartner.de/index.php?id=11070\&L=axdixyfwre 
Eine Sensitivität dieser $\mathrm{N}_{2} \mathrm{O}$ Emissionen ist ebenfalls gerechnet worden; die Ergebnisse werden unter [Arnold, 2010] veröffentlicht.

Nach Angaben von [KTBL, 2007] treten bei der Silierung des frischen Rohstoffs Verluste in der Größenordnung von 5 - 20 Massenprozent auf. Für den heute gängigen Stand der Technik wurde ein Erfahrungswert von $15 \%$ angesetzt. Für das Optimum wird ein Wert von $5 \%$ angesetzt, der sich - leicht konservativ - aus den nach [Ifeu, 2008] ,unvermeidbaren' Silierungsverlusten von $3-6 \%$ ableitet.

\section{Fermenter:}

Im Fermenter kann es bei der Umsetzung der Substrate zu Methan in Folge von Undichtigkeiten, Diffusion aus gasführenden Anlagenteilen oder aber durch Betriebsstörungen zu Leckagen kommen. Zur genauen Höhe dieser Leckagen besteht Forschungsbedarf, da hier noch keine exakten Messergebnisse vorliegen. Diese diffusen Methanemissionen aus der Biogasanlage selbst werden im Rahmen der Anforderungen an den Emissionsschutz, z.B. TA Luft, bislang kaum thematisiert [Landesumweltamt Brandenburg, 2008]. Hier wird bis zum Vorliegen genauerer Daten pauschal $1 \%$ der Methanproduktion analog zum Vorgehen in der Literatur [LFL, 2007] als Stand der Technik angesetzt. Als Annahme für ,Optimierte Technik' wird dieser Wert halbiert, um ein Optimum abzubilden. Es sei darauf hingewiesen, dass diese Annahmen bis zum Vorliegen genauerer Messdaten genutzt werden. Die Datenqualität ist in diesem Bereich derzeit leider deutlich geringer als für die anderen Angaben.

Ein weiterer wichtiger Parameter ist der Rohgasertrag, der angibt, wie viel Methan aus der eingebrachten Frischmasse (FM) erzeugt werden kann. Dies ist in weiten Teilen von der Art und Qualität des Substrats abhängig, aber auch die Ausführung des Fermenters und insbesondere der Gärvolumen hat einen Einfluss darauf ${ }^{8}$.. Bislang wird für Mais als Eingangsstoff nach Empfehlungen aus [FNR, 2006] mit einem Rohgasertrag für Mais von rund $200 \mathrm{~m} 3 / \mathrm{tFM}$ gerechnet. Erfahrung von Anlagenbetreibern zeigen aber bereits jetzt schon, dass um $10-18 \%$ höhere Gasausbeuten realisierbar sind. Für die Optimierte Technik -Anlage ist daher ein Wert von $220 \mathrm{m3} / \mathrm{t} \mathrm{FM}$ angesetzt worden.

\section{Gärrestmanagement:}

Beide hier abgebildeten Anlagenkonfigurationen orientieren sich an großtechnischen und modernen Biogasanlagen, die professionell betrieben werden. Aus diesem Grund wird für beide Anlagentypen ein vollständig abgedecktes Lager vorgesehen, bei dem die Methanemissionen durch Erfassung und Nutzung bzw. Abfackeln nahezu vollständig vermieden werden.

Indirekt emissionsrelevant ist auch der Stickstoffkreislauf, da je nach Anteil von direkt pflanzenverfügbarem Ammonium am Stickstoff mehr oder weniger große Mengen an Mineraldüngern substituiert werden. In Abhängigkeit von der Gasbildung (Ertrag und Methangehalt) wird auch der mit dem Substrat eingebrachte Stickstoff umgewandelt. Hauptprodukte sind dabei Ammoniak und Ammonium. Basierend auf Expertenschätzungen wird für die Anlage nach dem Stand der Technik ein Anteil von $60 \%$ an direkt pflanzenverfügbarem Ammonium am Stickstoff im Gärrest angenommen bzw. ein Anteil von 80 \% für die Optimierte Technik Anlage.

Der ausgebrachte Gärrest hat, wie beschrieben, einen Düngewert von 50 - $70 \%$ gegenüber dem mineralischen Stickstoffdünger [Gutser, 2008]. In der vorliegenden Arbeit ist mit einem mittleren Wert von $60 \%$ gerechnet worden. Die übrigen Nährstoffe, wie Phosphor, Kalium und Magnesium werden im Fermenter nicht abgebaut und können vollständig den Einsatz von mineralischem Dünger substituieren.

\section{Aufbereitung:}

Aus Gründen der Übersichtlichkeit wird an dieser Stelle mit der Pressure Swing Adsorption (PSA) nur ein Aufbereitungsverfahren exemplarisch behandelt. Von entscheidender Bedeutung ist der Methanverlust, der bei der Aufbereitung auftritt. Im seit Anfang 2009 novellierten EEG ist der zulässige Methanverlust auf 0,5 \% begrenzt [Landesumweltamt Brandenburg, 2008]. Nur mit dem PSA Verfahren kann diese Vorgabe nicht eingehalten werden, da hier typischerweise ein Wert von rund $2 \%$ erreicht wird [Fraunhofer UMSICHT, 2008]. Daher eine Nachverbrennung hinter die Aufbereitungsanlage geschaltet, in der das entweichende Methan entweder thermisch oder katalytisch umgesetzt wird (siehe ,Methanverlust' und ,Restemission' in Tabelle 1).

Da dieses Vorgehen den seit Januar 2009 geltenden Vorgaben entspricht, wird an dieser Stelle kein Unterschied zwischen den beiden Anlagenkonfigurationen gemacht. Der Methanverlust wird als stofflicher Verlust von $\mathrm{CH}_{4}$ in die Atmosphäre in die THG Bilanz einbezogen.

\section{Energiebedarf Biogasanlage und Aufbereitung:}

Wie in Abschnitt 2.1.1 beschrieben, wird zum Betrieb der Biogasanlage Energie in Form von Wärme zur Beheizung des Reaktors sowie Strom für Beschickung, Rührwerk etc. verbraucht. Auch die Aufbereitung (hier: PSA) muss mit elektrischer und thermischer Energie versorgt werden (vergl. Abschnitt 2.1.3). 
In Absprache mit den Teilnehmern des Expertenworkshops sowie dem Betreiber der hier dargestellten Anlage, optimierte Technik $^{9}$, sind die in Tabelle 1 aufgeführten Werte für den Energiebedarf der Biogasanlage angesetzt worden. Die Daten für die Aufbereitung sind [UMSICHT, 2008] entnommen. Für diese Werte wird keine Unterscheidung zwischen den beiden Anlagenkonfigurationen (Stand der Technik und Optimierte Technik) gemacht.

Im hier abgebildeten Fall wird die Energieversorgung beider Anlagenteile durch ein lokales wärmegeführtes BHKW $\left(\eta_{\mathrm{el}}=37,5 \% ; \eta_{\mathrm{th}}=43 \%\right)$ gewährleistet, dass einen Teil des erzeugten Biogases vor Ort unter Nutzung der Abwärme verstromt. Das benötigte Biogas steht damit nicht mehr zur Einspeisung zur Verfügung, so dass die Emissionen aus der Vorkette auf eine dann geringere Menge an eingespeistem Biomethan umgelegt werden und spezifisch ansteigen. Ein Vergleich mit der Option, Biogas nicht aufzubereiten und direkt vor Ort zu verstromen, ist unter anderem in [ifeu, 2008] und [Soukup, 2008] gerechnet worden. Die Ergebnisse sind stark von den jeweiligen Bedingungen insbesondere der Biogaserzeugung und Umwandlung der dezentralen Verstromung und der Wärmenutzung abhängig; es lässt sich aber festhalten, dass die Aufbereitung und Einspeisung von Biomethan ökologisch nicht nachteilig ist und zudem eine breitere Verwendung des erzeugten Energieträgers ermöglicht.

\subsubsection{Emissionsfaktoren der Biomethanprozesskette}

Abbildung 2 zeigt die Ergebnisse der THG Bilanz für die beiden Anlagentypen, bezogen auf TJ Biomethan an der Einspeisestelle. Den größten Anteil stellen - bei schon recht weit optimierter Anlagentechnik- die Emissionen aus der Substratbereitstellung, also die Aufwendungen für Düngemittel, Pflanzenschutz, Saatgut sowie Maschineneinsatz für die Aussaat, Düngung, Ernte und den Transport der Substrate zur Anlage. Dieser Abschnitt der Prozesskette ist in [Arnold\&Vetter, 2010] detailliert untersucht worden.

In etwa zu gleichen Teilen tragen die diffusen Emissionen aus dem Fermenter sowie die Energiebedarfsdeckung der Biogasanlage und Aufbereitung zur Klimabilanz bei. Bezüglich der Emissionen aus dem Fermenter sei an dieser Stelle noch mal auf die geringere Datenqualität hingewiesen, da bis zum Vorliegen von Messergebnissen mit Annahmen gearbeitet werden muss. Wird der Aufbereitung durch PSA eine Nachverbrennung hinzugeschaltet, wie es bei den abgebildeten Anlagen üblich ist, verringert sich der dann noch übrige Methanverlust mit 0,01\% auf so geringe Emissionen, dass diese in der Bilanz kaum noch erkennbar sind (vgl. Abbildung 2).

Abbildung 2: THG Faktoren der Prozesskette Biomethan (Basis: Nawaro): Vergleich der beiden Anlagenkonfigurationen (Aufbereitung: ca. 0,05 t/TJ bzW. 0,2 g/kWh)

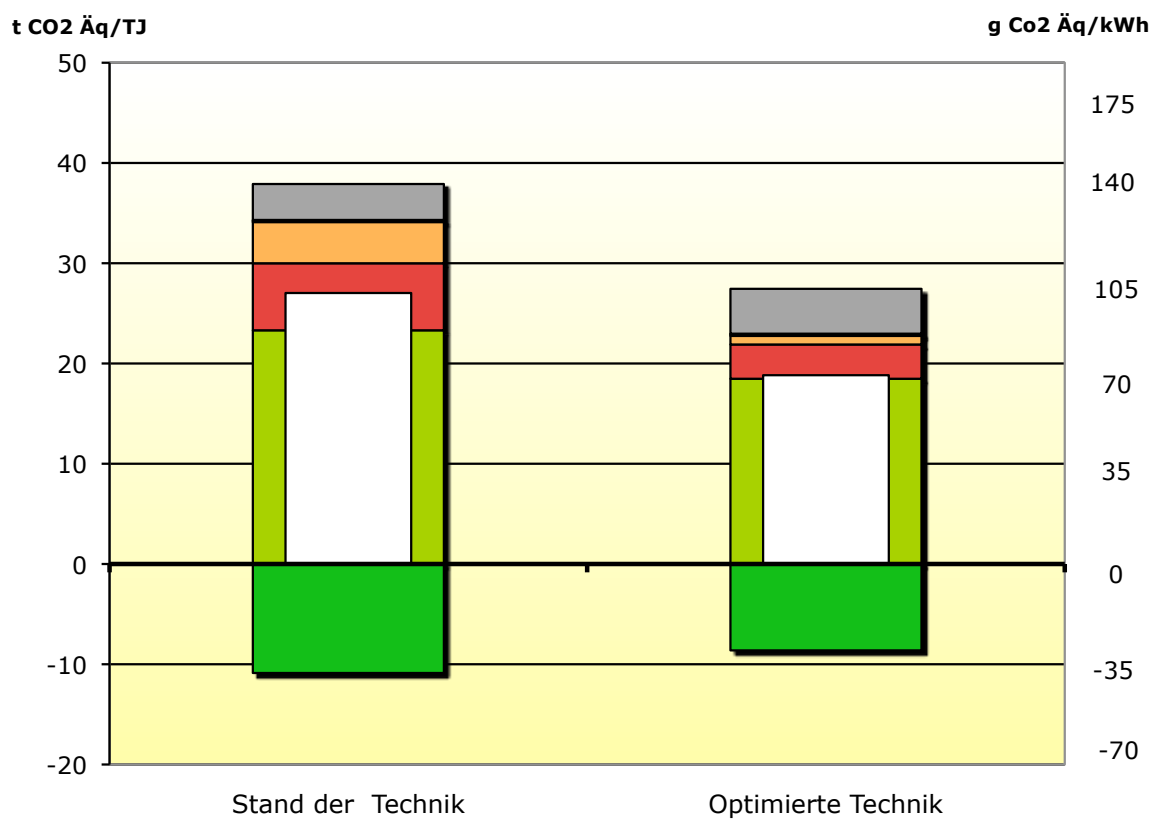

口Substratbereitstellung 口Aufbereitung 口Summe
口Gärrestmanagement $\square$ Gutschrift f. Düngung

9 als Referenz dient hier die Anlage Schwandorf II 
Obwohl beide Anlagen mit einem durchaus industriellen Ansatz betrieben werden, ist der Unterschied doch deutlich. Im Gegensatz zum derzeitigen Stand der Technik können mit wirklich optimierter Anlagenführung wie bei die der Anlage nach ,optimierter Technik' die THG Emissionen aus der Bereitstellung von Biomethan noch klar gesenkt werden.

Diese Differenz ist im Wesentlichen aus der Kombination aus höheren Silageverlusten und geringerem Rohgasertrag beim gegenwärtigen Stand der Technik zu erklären. Außerdem sind der höhere Methanverlust in der Biogasanlage sichtbar, sowie das schlechtere Stickstoffhandling bei der Ausbringung des Gärrests.

\subsection{Entwicklung der Biomethan-Prozesskette bis 2030}

Eine Dynamisierung der Anlagentechnik kann aus heutiger Sicht nur als vereinfachte Abschätzung bzw. grobe Tendenz erfolgen, da das erzielbare Maß an technischem Fortschritt nur schwer über einen Zeitraum von mehreren Dekaden absehbar ist.

Um dennoch zu einer Abschätzung des Optimierungspotenzials zu gelangen, werden die beiden untersuchten Anlagentypen ,Stand der Technik 2008' und ,Optimierte Technik' als Eckpfeiler angesetzt. Aus der Diskussion der technischen Parameter für den ,Optimierte Technik' Fall ist zuvor abgeleitet worden, dass die großen Emissionsminderungs- bzw. Optimierungspotentiale im Prozess der Biogaserzeugung damit bereits abgearbeitet sind. So sind z.B. die wesentlichen Methanquellen identifiziert und werden bei Anlagen nach ,Optimierte Technik' so weit wie derzeit möglich reduziert.

Weiteres Optimierungspotenzial liegt daher hauptsächlich noch im übergreifenden Prozessmanagement. So könnte z.B. die Abwärme der thermischen Nachbehandlung im Zuge der Aufbereitung zur Beheizung des Fermenters zurückgeführt werden. Eine Steigerung der Rohgasausbeute kann Auswirkungen auf die Fermenterbelastung und damit auf den Energiebedarf des Rührwerks mit sich ziehen. In wie weit das optimierte Zusammenspiel dieser einzelnen Komponenten sich auf die Effektivität der gesamten Anlage und damit auf die THG Bilanz niederschlägt, kann aber derzeit nicht im Detail vorausgesehen werden. Das mögliche Optimierungspotenzial wird daher als Fortschreibung der Verbesserung vom Anlagentyp ,Stand der Technik' zu ,Optimierte Technik' abgeschätzt.

Die Differenz in der THG Bilanz bei Berechnung der beiden Anlagenkonfigurationen beträgt ohne Anrechnung des Gärrests ca. $11 \mathrm{t} \mathrm{THG/TJ}$ oder $28 \%$, bei Rückführung des Gärrests ca. 8 t THG/TJ oder $30 \%$. Wie oben beschrieben, ist in dieser Differenz auch eine leichte Abweichung in der Datenqualität (bezüglich der diffusen Methanverluste im Fermenter) enthalten, was die Differenz vergrößert (Abbildung 3).

Abbildung 3: THG-Emissionsfaktoren für Biomethan (Basis Nawaro) zur Einspeisung aus den betrachteten Anlagen und Fortschreibung bis 2030 (eigene Darstellung)

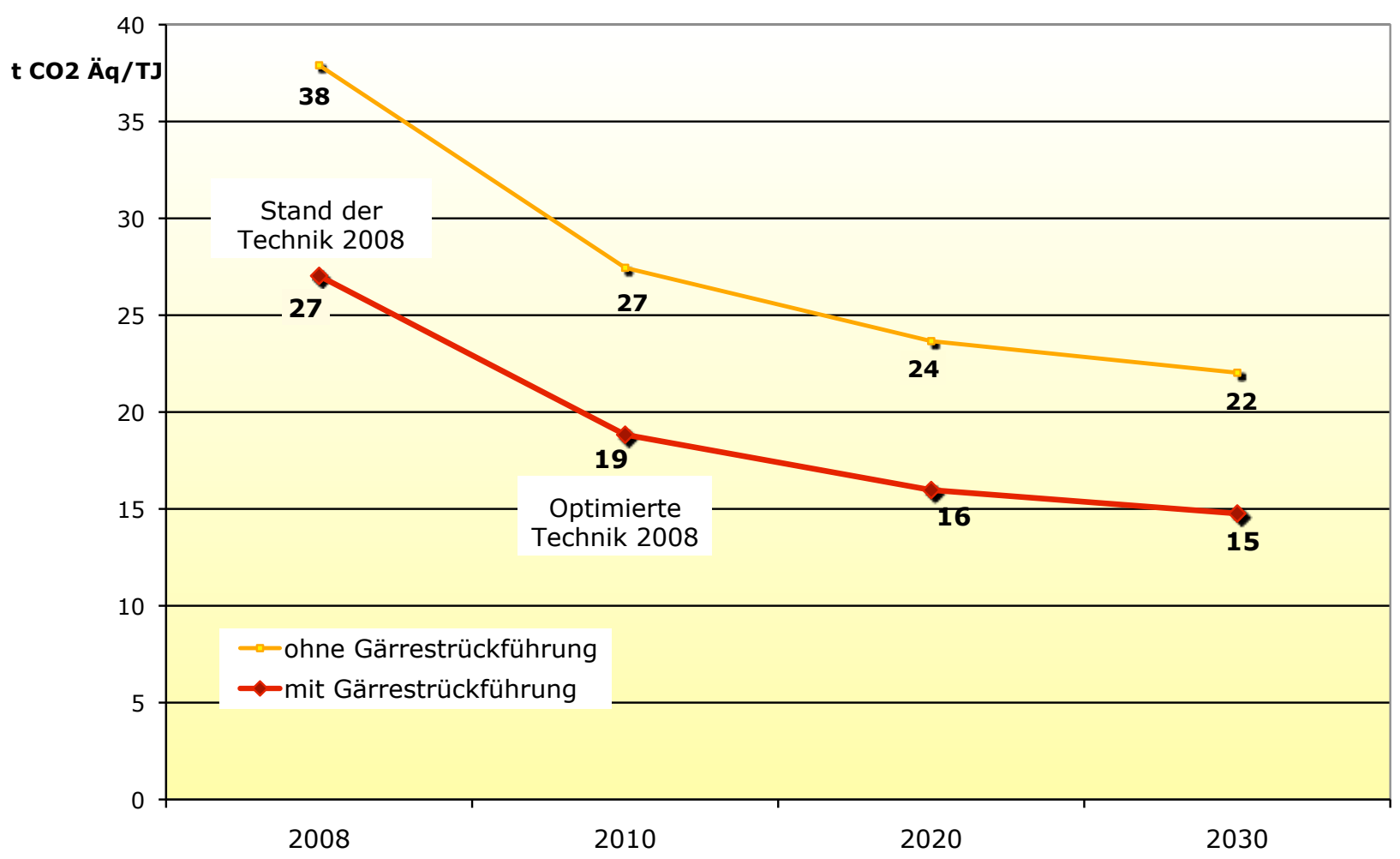

Es wird davon ausgegangen, dass ein ebenso großer Fortschritt bei der Entwicklung der nächsten Generation nicht wieder gelingt. Als Abschätzung wird daher angenommen, dass die nächste Generation eine Optimierung um die Hälfte erzielt. Gleiches gilt wiederum für die Dekade von 2020 - 2030. Die spezifischen Emissionen des Biomethans sinken demnach vom Ausgangspunkt (,Stand der Technik') mit rund 27 t THG/TJ bei Anrechnung des Gärrests auf 19 t THG/TJ für die ,optimierte Technik'. Für den Stützzeitpunkt 2020 kann mit spezifischen Emissionen von 16 t THG/TJ gerechnet werden; für 2030 mit nochmaliger leichter Verbesserung auf 15 t THG/TJ. 
Wenn die in Abbildung 4 angenommenen Mengen an Biomethan zukünftig zur Verfügung stehen sollen, wird ein erheblicher Zubau an großtechnischen Biogasanlagen notwendig. Unter dieser Voraussetzung kann angenommen werden, dass zum einen der oben abgeschätzte Fortschritt in der integrierten Anlagentechnik auch erzielt wird. Zum anderen können die ermittelten Werte als Annährung an einen Mittelwert der Prozesskette ,Biomethan zur Einspeisung' angesehen werden, da die vergleichsweise wenigen Anlagen, die heute mit weniger optimierter Technik betrieben werden (,Stand der Technik 2008') dann in der Gesamtbetrachtung kaum ins Gewicht fallen.

\section{Erdgasprozesskette}

Erdgas stammt aus unterirdischen Lagerstätten und tritt über Bohrlöcher an die Erdoberfläche. Es wird je nach Konsistenz aufbereitet und falls notwendig komprimiert ${ }^{10}$. Da viele für Deutschland relevante Fördergebiete, wie z.B. die westsibirischen Vorkommen, weit entfernt liegen, wird das Erdgas durch Hochdruckleitungen über weite Distanzen transportiert. Hierfür werden ca. alle 100-200 km Verdichterstationen benötigt, die das Gas nach Druckabfall wieder auf ca. 80 bar komprimieren und dazu in der Regel einen Teil des Gases als Betriebsenergie einsetzen. In der Zielregion wird das Gas dann mit entsprechend geringerem Druck über regionale Mittel- und Niederdrucknetze verteilt. Eine weitere Aufbereitung ist nicht mehr von Nöten. Das Erdgas kann beim Endnutzer, sei es Industrie oder Haushalt, direkt eingesetzt bzw. verbrannt werden.

\subsection{Treibhausgasemissionen der Erdgas-Prozesskette}

Potentiell werden bei allen Prozessschritten der Erdgas-Prozesskette THG-Emissionen freigesetzt, d.h. bei der Förderung und Gasaufbereitung, beim Transport sowie bei der Gasverteilung bis zum Endverbraucher. $\mathrm{Zu}$ unterscheiden ist hier zwischen den $\mathrm{CO}_{2}$-Emissionen und $\mathrm{CH}_{4}$-Emissionen. Erstere entstehen bei der Verbrennung des Erdgases vor allem in den Turbinen der Verdichterstationen. $\mathrm{CH}_{4}$-Emissionen entstehen, wenn Erdgas über technologisch bedingte Freisetzungen, z.B. bei Wartungsarbeiten, oder über Leckagen und Betriebsstörungen in die Atmosphäre gelangt. Dabei weist Methan als Hauptbestandteil des Erdgases unverbrannt ein um das 21 bis 25 fache höheres Treibhausgaspotential ${ }^{11}$ als $\mathrm{CO}_{2}$ auf.

Die tatsächlichen Emissionen der Gasförderung und -aufbereitung hängen stark von den individuellen Bedingungen vor Ort ab. Günstig sind dabei z.B. hohe Methankonzentrationen und ein hoher natürlicher Druck des Feldes, so dass das Gas weder komprimiert werden muss, um die ersten Kilometer in der Pipeline zurückzulegen noch eine zusätzliche Aufbereitung erforderlich ist. Die Emissionen des Gasferntransports sind entscheidend von der Transportdistanz, den Wartungsbedingungen sowie dem Zustand der Kompressorstationen und der Pipelines abhängig. [vgl. Wuppertal Institut 2005; Lechtenböhmer\&Dienst 2008].

Im Vergleich zu dem durch Pipelines transportierten Erdgas nimmt das verflüssigte Erdgas (Liquified Natural Gas) eine Sonderrolle ein. Es durchläuft auf seinem Weg bis zum Endverbraucher drei zusätzliche Prozessschritte: 1. die Verflüssigung durch Abkühlung, 2. die Speicherung und den Schiffstransport sowie 3. die Regasifizierung. Alle drei Schritte benötigen Energie, was zu CO 2 -Emissionen führt, und sind weitere Quellen flüchtiger Methanemissionen [vgl. Lechtenböhmer\&Dienst 2008].

\subsection{Entwicklung der Erdgasprozesskette bis 2030}

In den kommenden Jahrzehnten wird sich der Erdgas-Importmix Deutschlands und weltweit erheblich verändern. Da sich die Prozesskettenemissionen je nach Förderregion und Transportweg deutlich unterscheiden, wird dies auch Auswirkungen auf die damit verknüpften THG-Emissionen mit sich bringen. Außerdem wird sich die Technik der Förderung und des Transports - und damit die spezifischen Emissionen -, vor allem in Russland, verändern.

\subsubsection{Szenarien emissionsrelevanter Veränderungen der (russischen) Erdgasinfrastruktur}

Die russische Gasförderung wird sich in den kommenden Jahrzehnten deutlich diversifizieren. Die bisher hauptsächlich für den Export bestimmten Super-Giant Felder Urengoy, Jamburg und Medwezhe haben ihr Fördermaximum überschritten und ihr Anteil an der russischen Erdgasförderung wird kontinuierlich sinken. Stattdessen werden tiefere Förderschichten und vor allem neue kleinere on-shore und off-shore Felder von Gazprom und unabhängigen Gesellschaften sowie durch die Ölindustrie erschlossen werden. Dazu kommt die geplante Erschließung größerer Felder auf der Yamal-Halbinsel und des

10 Erdölbegleitgas muss in der Regel aufbereitet werden. In reinen Erdgasfeldern wird meist trockenes Erdgas gefördert, das häufig direkt ohne Kondensatabscheidung abgekühlt und genutzt werden kann.

11 Der ,Fourth Assessment Report' des IPCC (IPCC 2007) gibt für Methan die 25-fache Treibhausgaswirkung im Vergleich zu CO $\mathrm{CO}_{2}$ und bezogen auf einen Zeitraum von 100 Jahren an. Im vorherigen Assessment Report wurde ein Treibhausgaspotential von 23 angegeben (IPCC 2001). Aktuell rechtlich bindend für die mit dem Kyoto-Protokoll zusammenhängenden Belange, d.h. bis 2012, ist der ,Second Assessment Report' (IPCC 1995), in dem das Treibhausgaspotential auf 21 festgelegt wurde. Daher ist in den Berechnungen bei Lechtenböhmer\&Dienst (2008) durchgängig der Wert von 21 verwendet worden. Gemis 4.4 (Öko-Institut 2006) verwendet ein THG-Potential von 23 für Methan. Hier werden dagegen durchgängig die aktuellen Werte des 4th Assessment Reports verwendet, da davon ausgegangen werden kann, dass diese Werte ab 2012 auch rechtlich verbindlich sein werden. 
off-shore Felds Stockman in der Barentssee. Außerdem wird erwartet, dass der Import von Erdgas aus Turkmenistan sowie Kasachstan und Usbekistan stark ansteigt [vgl. Lechtenböhmer \& Dienst 2008, basierend auf IEA 2006a, 2006b, 2005, 2004, CIEP 2004, IGU 2006, Medvedev 2006, Stern 2006, SWP 2006].

Vor diesem Hintergrund analysieren Lechtenböhmer\&Dienst [2008] mögliche Modernisierungs- und Investitionspfade der russischen Gasinfrastruktur. Aus unserer Sicht ist demnach zu erwarten, dass die zukünftige Emissionssituation der russischen Erdgaswirtschaft von zwei gegenläufigen Trends bestimmt wird:

- Die Zahl der fördernden Felder und Bohrungen wird aufgrund sinkender Feldgrößen und geringere Förderung pro Bohrloch stark ansteigen, was zu tendenziell steigenden spezifischen Emissionen führen wird.

- Gleichzeitig erwarten wir, dass die technische Infrastruktur sowohl im Bereich der Fördertechnik als auch in neu gebauten und modernisierten Pipelines deutlich verbessert wird. Denn die erforderlichen umfangreichen Neuinvestitionen werden nach dem heutigen Stand der Technik durchgeführt werden und damit zu einer klaren Senkung der spezifischen Emissionen beitragen.

Insgesamt gehen Lechtenböhmer\&Dienst [2008] davon aus, dass die spezifischen Treibhausgasemissionen aus Transportenergieaufwand und Leckagen - bezogen auf die frei deutscher Grenze gelieferte Energiemenge - im Vergleich zu heutigen Werten, je nach der Geschwindigkeit der Reinvestitionen und dem dabei verwendeten technischen Standard bis 2030 um 30 bis $50 \%$ sinken werden.

Auch für die übrigen Förderregionen wird aufgrund technischer Verbesserungen von einer Verringerung der Vorkettenemissionen um 12 - 30\% pro gelieferter Energieeinheit ausgegangen (siehe Tabelle 2; Öko-Institut 2006).

\subsubsection{Treibhausgasemissionen entlang der Erdgasprozesskette bis 2030}

Die Erdgasprozesskette für Endkunden in Deutschland setzt sich aus verschiedenen emissionsrelevanten Komponenten zusammen, der fossilen bzw. der biogenen Prozesskette bis zur Einspeisung in das inländische Netz, der regionalen und lokalen Verteilung und schließlich der Verbrennung beim Endnutzer.

- Tabelle 2 zeigt zunächst die mit Förderung und Aufbereitung in den jeweiligen Herkunftsländern sowie dem Ferntransport nach Deutschland verbundenen spezifischen, d.h. auf die frei deutscher Grenze gelieferte Energieeinheit bezogenen, Treibhausgasemissionen. Diese unterscheiden sich, vor allem aufgrund der eingesetzten Technik aber auch der Entfernung, deutlich. Infolge von Re-Investitionen in verbesserte Technik werden sich die Emissionsfaktoren für Gasimporte aus den verschiedenen Herkunftsregionen in der Zukunft z.T. deutlich verändern. Hierbei sind für Russland nach Lechtenböhmer \& Dienst [2008] zwei verschiedene Varianten angenommen worden.

- Die auffällig hohen THG-Emissionen die mit der Einfuhr des verflüssigten Erdgases verbunden sind, sind auf die beschriebenen zusätzlichen Prozessschritte zurück zu führen. Die Emissionen von LNG liegen aktuell mit 12,8 t $\mathrm{CO}_{2}$ - $\mathrm{A} . / \mathrm{TJ}$ in etwa dreimal höher als die Emissionen für den Import norwegischen Erdgases. Hier ist aufgrund der bislang noch sehr begrenzten Datenverfügbarkeit eine konservative, d.h. hohe Abschätzung vorgenommen worden. Entsprechend des künftig verstärkten Ausbaus dieser recht jungen Technik wird hier auch ein hohes Verbesserungspotential um $40 \%$ auf 7,8 t CO 2 - $\mathrm{Ä}$./TJ gesehen. Diese Werte sind als grobe Kennwerte zu betrachten, die dem Gemis-Model entnommen wurden [Öko-Institut 2006]. Der Energieaufwand für die Verflüssigung des Erdgases nimmt ca. 70\% der spezifischen THG-Emissionen der Vorkette ein. Daneben sind 4-5\% der Emissionen auf den Schiffstransport zurückzuführen, inklusive Be- und Entladung und Verdampfung. Der Rest ist bedingt durch die normale Erdgasförderung und -aufbereitung [siehe auch Lechtenböhmer\&Dienst 2008].

- Ebenso sind die vorgelagerten Emissionen der, künftig zunehmenden, Biogaseinspeisung zu berücksichtigen. Bezogen auf die eingespeiste Menge werden sie ebenfalls durch technische Verbesserungen zurückgehen.

- Dritte vorgelagerte Emissionsquelle ist die regionale und lokale Verteilung in Deutschland, bei der nach Angaben von von E.ON-Ruhrgas ca. $0,5 \%$ des Gases emittieren ${ }^{12}$.

- Quantitativ dominierend gegenüber den Vorkettenemissionen sind die direkten Emissionen der Verbrennung des fossilen Erdgases. Die bei der Verbrennung von Biomethan entstehenden $\mathrm{CO}_{2}$-Emissionen werden nicht eingerechnet, da es sich hier um biogenen Kohlenstoff handelt, der lediglich dem natürlichen Kohlenstoffkreislauf wieder zugeführt wird.

Entscheidend für die spezifischen Emissionen der Erdgasnutzung in Deutschland ist - neben den technischen Verbesserungen in den Lieferländern - der Mix der verschiedenen Erdgasquellen. Um diese Effekte adäquat berücksichtigen zu können werden im folgenden Abschnitt zwei Szenarien des möglichen künftigen Gaseinsatzes in Deutschland und seiner Bereitstellung dargestellt.

12 Gemis geht von Verteilnetzverlusten von 0,4\% aus [Öko-Institut 2006]; laut Angaben von E.ON-Ruhrgas belaufen sich die Verluste auf 0,5\%. Dieser Wert wurde für die Berechnungen konstant für die Verteilverluste in Deutschland bis 2030 eingesetzt. 
Tabelle 2: Indirekte und direkte THG-Emissionsfaktoren von Erdgas und Biomethan, differenziert nach Lieferländern für Deutschland (2005 - 2030)

\begin{tabular}{|c|c|c|c|c|}
\hline In t $\mathrm{CO}_{2} \mathrm{Ä} . / \mathrm{TJ}$ & Referenz* & 2020 & 2030 & $\begin{array}{l}\text { Minderung ggb. } \\
\text { Referenz }\end{array}$ \\
\hline \multicolumn{5}{|l|}{$\begin{array}{l}\text { Vorkettenemissionen fossiles Erdgas } \\
\text { (nach Lieferland frei deutscher Grenze/Einspeisepunkt) }\end{array}$} \\
\hline \multicolumn{5}{|l|}{ Russland } \\
\hline Hoher Verbrauch + hohe Investitionen & 15,6 & & 6,8 & $56 \%$ \\
\hline Niedriger Verbrauch + niedrige Investitionen & & 12,3 & 11,1 & $29 \%$ \\
\hline LNG (Algerien, Katar, Ägypten) & 12,9 & 8,6 & 8,0 & $38 \%$ \\
\hline Norwegen & 3,9 & 3,6 & 3,2 & $18 \%$ \\
\hline Niederlande & 2,7 & 2,3 & 1,9 & $44 \%$ \\
\hline GB/DK ${ }^{a}$ & 3,9 & 3,6 & 3,2 & $18 \%$ \\
\hline D Inland Erdgas & 2,7 & 2,6 & 2,4 & $11 \%$ \\
\hline \multicolumn{5}{|l|}{ Vorkettenemissionen Biomethan } \\
\hline D Biomethan (mit Gärrestrückgewinnung) & 27,0 & 16,0 & 14,8 & $45 \%$ \\
\hline \multicolumn{4}{|l|}{ Weitere THG-Emissionen: } & \\
\hline Verteilung in Deutschland $^{b}$ & 2,4 & 2,4 & 2,4 & \\
\hline Direkte THG-Emissionen von fossilem Erdgas & 56,0 & 56,0 & 56,0 & \\
\hline Direkte THG-Emissionen von Biomethan & $\begin{array}{r}\text { Da der K } \\
\text { Atmosphäre }\end{array}$ & $\begin{array}{l}\text { ff bei Bic } \\
\text { wurde } \\
\text { ssionen }\end{array}$ & $\begin{array}{l}\text { vor der } \\
\text { ne direkten }\end{array}$ & \\
\hline
\end{tabular}

Quelle: eigene Annahmen und Kennwerte von Gemis 4.4 [Öko-Institut 2006], berechnet mit GWP für Methan von 25; a: gleichgesetzt mit Emissionsfaktoren Norwegen; *Kennwerte von 2003 für Russland, sonst 2005; b: 0,5\% des verbrauchten Gases (nach E.ON Ruhrgas, in Öko-Institut 2006 werden 0,4\% genannt); Um die Emissionen frei Endnutzer in Deutschland zu berechnen müssen die indirekten Emissionen des jeweiligen fossilen oder biogenen Gases zu den Emissionen der Verteilung und (für fossiles Gas) den direkten Emissionen bei der Verbrennung addiert werden. Im Folgenden werden entsprechende Mittelwerte für den Gasmix in Deutschland abgeleitet.

\section{Zukünftige THG-Emissionen der Gasversorgung in Deutschland}

Die Vorkettenemissionen des in Deutschland genutzten Gases unterscheiden sich deutlich, je nach Herkunft. Um zu Aussagen zum in Deutschland eingesetzten Gasmix zu kommen, werden daher zunächst Szenarien der künftigen Gasnachfrage sowie Gasbeschaffung entwickelt. Dabei werden zwei Szenarien unterschieden, ein Hochverbrauchsszenario, das bisherige Trends fortschreibt ${ }^{13}$ sowie ein Niedrigverbrauchsszenario, das davon ausgeht, dass es im Rahmen von Klimaschutzstrategien zu einem Rückgang der Gasnachfrage kommt (siehe Abb. 4).

Diese beiden Nachfrageszenarien haben jeweils spezifische Auswirkungen auf die Lieferstruktur des Gases. Die hierzu getroffenen Annahmen berücksichtigen Experteneinschätzungen aus der Gaswirtschaft und Prognosen von ,Global Insight' [2005].

- Für die kommenden Jahrzehnte wird unabhängig vom Verbrauch erwartet, dass die Importe aus den EU-Ländern gen Null tendieren, d.h. Gas aus den Niederlanden, Großbritannien und Dänemark wird fast keine Rolle mehr für den deutschen Markt spielen. Ebenso wird die Förderung deutschen Erdgases von heute ca. 16 Mrd. $\mathrm{m}^{3}$ auf 3-4 Mrd. $\mathrm{m}^{3}$ sinken.

- Diese sinkenden Liefermengen werden zu einem mehr oder weniger großen Teil durch neue Quellen ersetzt. Dies sind Biomethan für das ein Anstieg der Produktion und Einspeisung bis 2020 auf 6 Mrd. $\mathrm{m}^{3}$ und bis 2030 auf 10 Mrd. $\mathrm{m}^{3}$ politisch angestrebt wird [BMWI 2006] ${ }^{14}$ und LNG, das im Hochverbrauchsszenario aus Algerien und anderen Ländern wie z.B. Ägypten stammen wird.

- Verflüssigtes Erdgas und Biomethan werden im Niedrigverbrauchsszenario die Bezugslücken in Höhe von ca. 25 bis $30 \%$ durch den Fortfall der EU-Förderung weitgehend decken können. Im Hochverbrauchsszenario dagegen werden die Bezugsmengen aus den Hauptlieferländern Norwegen und Russland weiter ansteigen.

13 Der über ca. 2 Jahrzehnte anhaltende Anstieg des Erdgaseinsatzes hat sich in den letzten Jahren allerdings deutlich abgeschwächt. Deshalb ist dieses Szenario eher als Abschätzung einer möglichen oberen Bandbreite zu verstehen.

14 Diese Mengen liegen leicht über den Schätzungen die in der Vorgängerstudie verwendet wurden [Lechtenböhmer\&Dienst 2008]. 
Abbildung 4: Gasnachfrage und -versorgung: 2 Szenarien für Deutschland bis 2030

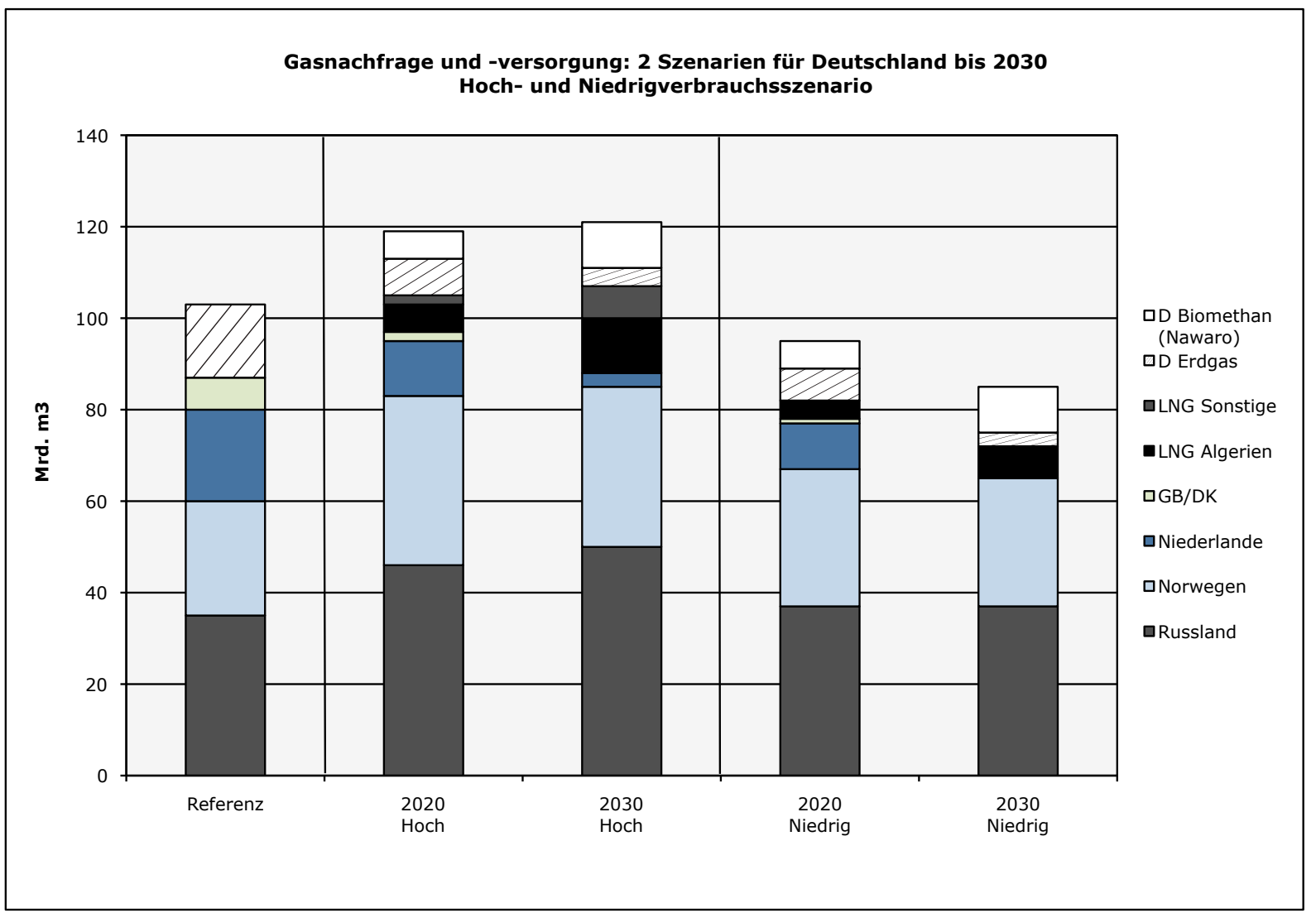

Quelle: eigene Schätzungen, basierend auf Lechtenböhmer\&Dienst [2008] nach EWI [2005], BMU [2004], Global Insight [2005] und BMWi [2006]

\subsection{THG-Emissionen des Gaseinsatzes bis 2030}

Im Jahr 2005 wurden in Deutschland insgesamt ca. 103 Mrd. $\mathrm{m}^{3}$ Erdgas eingesetzt. Die direkten Emissionen der Verbrennung lagen - entsprechend eines Emissionsfaktors von $56 \mathrm{t} \mathrm{CO}_{2} / \mathrm{TJ}$ - bei rd. $183 \mathrm{Mio}$. t $\mathrm{CO}_{2}$. Indirekt waren mit diesem Verbrauch weitere Treibhausgasemissionen in Höhe von 24,4 Mio. t CO 2 -Äquivalenten verbunden, die zum Großteil (70\%) aus der Förderung und dem Transport des westsibirischen Erdgases stammen, gefolgt von den Emissionen aus der Prozesskette des norwegischen Erdgases (ca. 13\%) (vgl. Tabelle 3). Das ist v.a. dem hohen Anteil beider Herkunftsländer am Gasmix und den weiten zu überbrückenden Transportwegen geschuldet. Durch den Energieaufwand des Transports und Methanemissionen bei der Verteilung in Deutschland wurden weitere 7,9 Mio. t $\mathrm{CO}_{2}$-Äquivalenten freigesetzt.

Da die Treibhausgasemissionen des Gaseinsatzes durch die Verbrennung dominiert werden, wird der absolute Einsatz fossilen Erdgases auch in Zukunft die wichtigste Treibergröße für die Emissionen sein. Jedoch werden der Anteil des Biomethans und die Entwicklung der Vorkettenemissionen die Gesamtemissionen zunehmend beeinflussen.

- Im Hochverbrauchsszenario werden die Emissionen bis 2030 anstatt um 17\% - wie der Gasverbrauch - nur um 7\% steigen (auf 230,9 Mio. t $\mathrm{CO}_{2}-\ddot{A}$.). Das ist bedingt durch die erheblichen technischen Verbesserungen bei der Erdgasinfrastruktur sowie die durch den verstärkten Einsatz von Biomethan vermiedenen direkten Verbrennungsemissionen. Aufgeschlüsselt nach Herkunft der indirekten Emissionen, wird im Vergleich zu heute eine Verschiebung deutlich. Die Emissionen der russischen Erdgaskette tragen nur noch knapp 44\% der indirekten Emissionen bei, gefolgt von den Emissionen von LNG mit 20\% und denen der Biomethankette mit 19\%.

\begin{tabular}{|c|c|c|c|c|c|}
\hline $\begin{array}{l}\text { Tabelle 3: THG-Emissionen der Vorkette der Gasversorgung in Deutschland - heute bis } 2030 \\
\text { In Mio. } \mathbf{t} \mathrm{CO}_{2} \mathrm{~A} \text {. }\end{array}$ & 2005 & 2020 & 2030 & 2020 & 2030 \\
\hline \multicolumn{2}{|l|}{ Vorkettenemissionen fossiles Erdgas * } & \multicolumn{2}{|c|}{ Hochverbrauchsszenario } & \multicolumn{2}{|c|}{ Niedrigverbrauchsszenario } \\
\hline Gesamt Vorkette Gasversorgung* & 24,4 & 23,5 & 24,2 & 23,5 & 22,5 \\
\hline \multicolumn{6}{|l|}{ THG-Emissionen der Verbrennung und Verteilung in $D$} \\
\hline Verteilung in $D$ & 7,9 & 9,2 & 9,3 & 7,3 & 6,6 \\
\hline Direkte Emissionen der Verbrennung & 183,1 & 200,1 & 197,3 & 158,2 & 133,3 \\
\hline Gesamtemissionen direkt und indirekt & 215,4 & 233,5 & 230,9 & 189,0 & 162,4 \\
\hline
\end{tabular}

*bis Grenze bzw. KW ohne Gasverteilung im deutschen Gasnetz; **) Einspeisemengen in beiden Szenarien gleich angenommen. 
- Im Niedrigverbrauchsszenario werden die absoluten THG-Emissionen bis 2030 infolge des Verbrauchsrückgangs und durch den verstärkten Einsatz von Biomethan erheblich von 215,4 auf 162,4 Mio. t $\mathrm{CO}_{2}-\ddot{A}$. sinken. Das entspricht einem Rückgang der Emissionen von 25\% im Vergleich zu einem Rückgang des Gasverbrauchs um nur 17\%. Allerdings ist bei den darin enthaltenen indirekten Emissionen nur eine leichte Senkung abzusehen (von 24,4 auf 22,5 Mio. t $\mathrm{CO}_{2}$ - $\mathrm{A}$.; vgl. Tabelle 3). Das ist einerseits auf die in diesem Szenario niedrigeren Investitionen und damit langsameren technischen Optimierungen der russischen Gasinfrastruktur zurückzuführen und andererseits auf den höheren Anteil von Biomethan. Mit einem Anteil von 12\% am gesamt genutzten Gas werden die Emissionen der Biomethanvorkette ca. 21\% zu den indirekten Emissionen beitragen.

Die Abbildungen 5 und 6 fassen die erläuterten Ergebnisse der Szenarienberechnung komprimiert zusammen. Erstere schlüsselt die vorgelagerten Emissionen der Vorketten nach Herkunft des Gases auf. Obwohl sich die Anteile gemäß der veränderten Lieferstruktur verändern, schwanken die Gesamtmengen nur leicht. Abbildung 6 stellt das Gesamtbild der THGEmissionen der deutschen Gasversorgung dar und verdeutlicht den Einfluss des Biomethaneinsatzes auf die gesamten Emissionen, inklusive der vermiedenen direkten Emissionen durch die Verbrennung.

Abbildung 5: Entwicklung der THG-Emissionen der Vorketten von Erdgas und Biomethan, bezogen auf den gesamten Gaseinsatz in Deutschland $(2005-2030)$

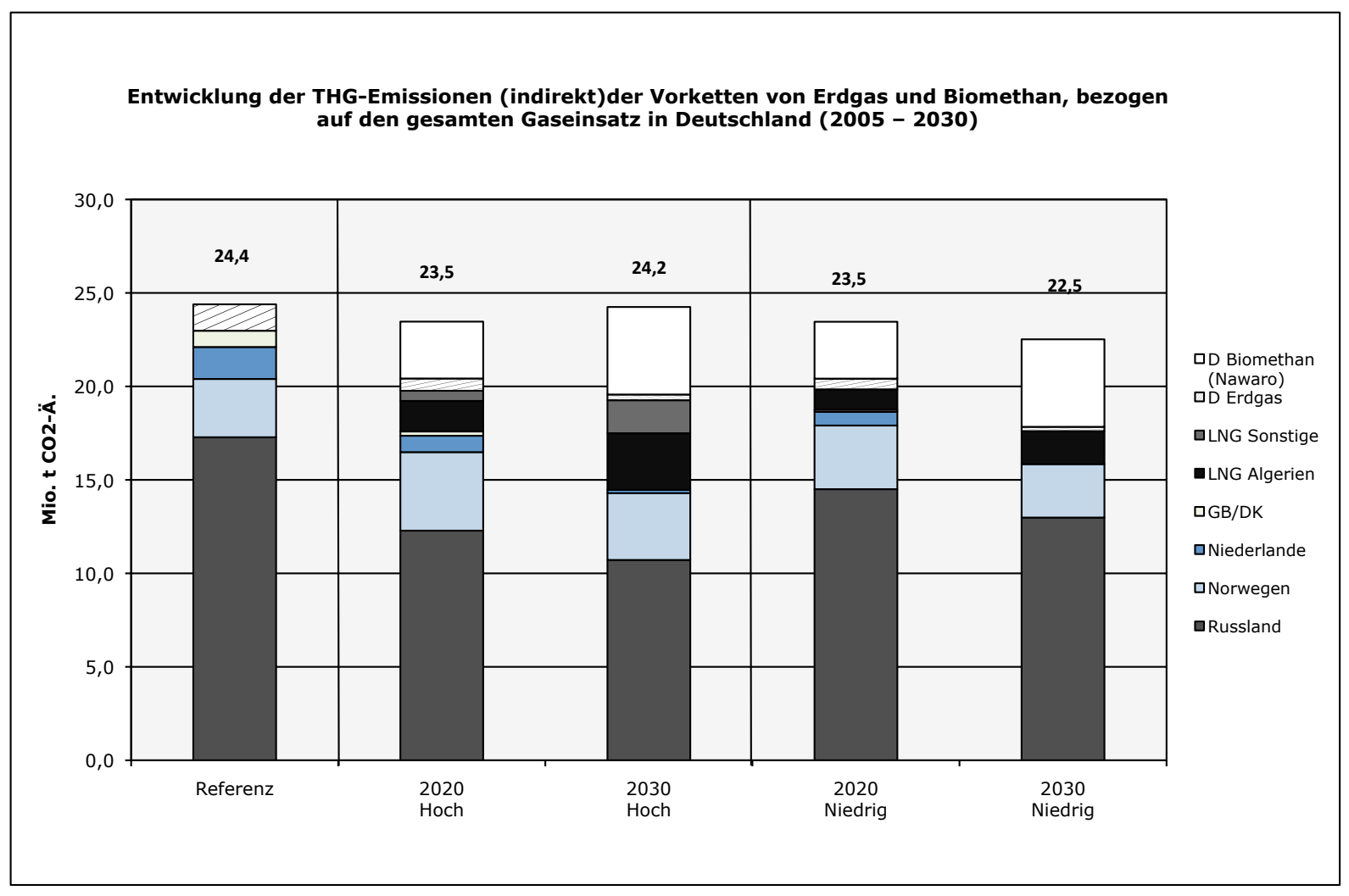

\subsection{Einordnung im Vergleich zu anderen fossilen Energieträgern}

Im Vergleich zu den anderen fossilen Energieträgern bleiben die gesamten THG-Emissionen des Erdgases und zukünftigen Gasmixes auf einem niedrigen Niveau. Bereits die direkten Treibhausgasemissionen der Verbrennung von Steinkohle und Braunkohle und Mineralölprodukten liegen mit 93 t/TJ, 112 t/TJ bzw. 78 t/TJ deutlich oberhalb der Gesamtemissionen des deutschen Erdgasmixes - einschließlich der Vorkettenemissionen. Hinzu kommen bei Kohle und Ölprodukten ebenfalls noch die Emissionen entlang der Vorketten. Für Heizöl (HEL) hängt ihre zukünftige Entwicklung wie beim Erdgas von der Entwicklung des Zuliefermixes und den anstehenden technischen Veränderungen in den jeweiligen Förderländern ab. Nach Schätzungen der Prozesskettenmodelle werden im Bereich der Förderung und des Transports erhebliche Verbesserungen der Technik bzw. eine Angleichung an , best available technology' erwartet. Dies ist insbesondere für die Situation der Erdölindustrie in Russland zu erwarten (Öko-Institut 2006). Im emissionsrelevantesten Bereich der Prozesskette, der Raffinierung in Deutschland, kann man dagegen von einem gleichbleibenden Emissionsniveau ausgehen, da hier die Standards bereits als hoch einzuschätzen sind [JRC 2003]. 
Abbildung 6: Mittlere THG-Emissionen des in Deutschland genutzten Erdgas und Biomethan-Mixes (2005 bis 2030$)$ in t $\mathrm{CO}_{2}-\mathrm{Äquivalent/TJ}$ im Vergleich zu den THG-Emissionen anderer fossiler Energieträger

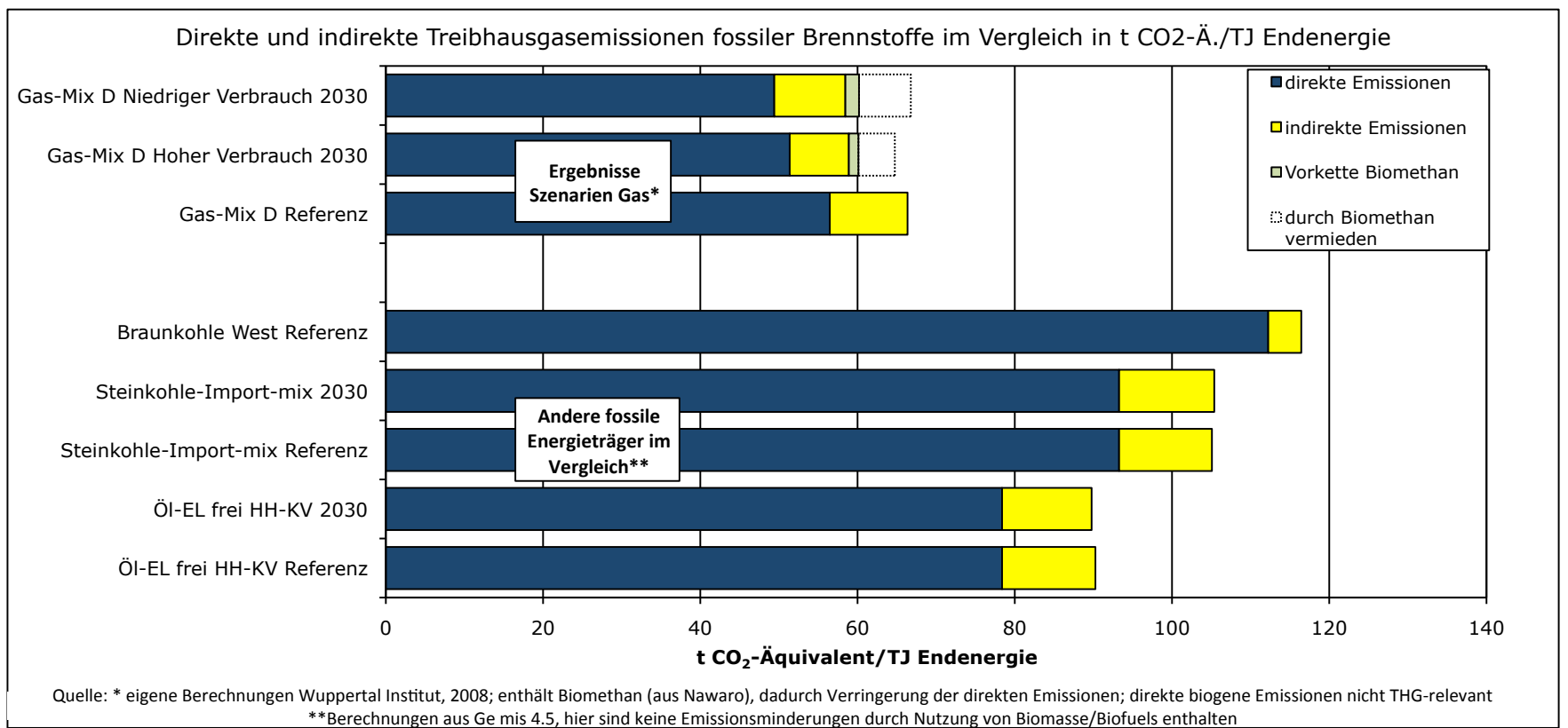

Die direkten $\mathrm{CO}_{2}$-Emissionen bei der Verbrennung von Biomethan werden in der Treibhausgasbilanz nicht berücksichtigt, da sie aus vorher beim Aufwuchs der Biomasse aus der Atmosphäre gebundenen Kohlenstoff stammen. Die im Verbrennungsprozess ebenfalls entstehenden Emissionen von $\mathrm{CH}_{4}$ und $\mathrm{N}_{2} \mathrm{O}$ sind marginal und werden daher hier nicht berücksichtigt.

Gasförmige Energieträger werden in den kommenden beiden Dekaden weiterhin eine zentrale Säule der deutschen Energieversorgung bleiben. Dabei wird sich das Gas in energiepolitischer Hinsicht bezüglich seiner Herkunft sowie in klimapolitischer Hinsicht bezüglich seiner Klimaqualität - d.h. der Gesamtheit der über die Prozesskette mit seiner Nutzung verbundenen Treibhausgasemissionen - deutlich weiterentwickeln. Es wird dabei drei entscheidende Veränderungstendenzen geben: Die Veränderung der Lieferstruktur des fossilen Erdgases, die Verbesserungen der Gasinfrastrukturen sowie die zunehmende Einspeisung von Biomethan in das deutsche Gasnetz.

Die Bezugsstruktur von Erdgas wird sich in den kommenden beiden Dekaden weiterentwickeln, weg vom Erdgas aus der EU, deren Gasfelder nahezu erschöpft sind, und stärker hin zu den bereits heute zentralen Lieferanten in Russland und Norwegen. Als neue Bezugsquelle kommt verflüssigtes Erdgas (LNG) hinzu.

Je nach Fördergebiet existieren bei den Förder- und Transportinfrastrukturen unterschiedlich hohe Optimierungspotentiale. Insbesondere für die sich stärker diversifizierende russische Erdgasförderung ist je nach Investitions- und Modernisierungsgrad eine deutliche Verbesserung der Emissionsfaktoren möglich. Das hat entscheidenden Einfluss auf die Vorkettenemissionen des in Deutschland verbrauchten Gases, da bis 2030 fast die Hälfte des Gases aus Russland stammen wird. Insgesamt können - trotz einer emissionsseitig ungünstiger werdenden Bezugsstruktur - können die Vorkettenemissionen des fossilen Gasmixes in Deutschland durch eine Verbesserung der eingesetzten Technik von heute durchschnittlich ca. 7 t CO $2-\ddot{A} . / T J$ auf 6,3 bis 4,9 t CO $2-\ddot{A} . / T J$ (ohne Gasverteilung in Deutschland) verringert werden.

Ein weiterer wichtiger Trend wird die Einspeisung inländisch erzeugten Biomethans in das deutsche Gasnetz sein, das im Jahr 2030 voraussichtlich einen Anteil von bis zu $8-12 \%$ des Gesamtverbrauch abdecken kann. Die zukünftigen Treibhausgasemissionen der noch jungen Technik der Biomethanproduktion hängen ebenfalls zu einem Großteil von den technischen Entwicklungen und den künftigen Investitionen in neue bzw. erneuerte Infrastrukturen ab.

- In sämtlichen Prozessschritten, der Substratbereitstellung, der Fermentierung und der Aufbereitung, entstehen $\mathrm{CO}_{2}$ Emissionen durch den Energiebedarf an Strom und Wärme. Zusätzlich treten Methan und Lachgas über Leckagen oder Ausgasungen aus, was aufgrund der hohen Treibhausgaspotentiale dieser Gase bedeutend für die Emissionsbilanz ist. Andererseits kann die Substitution von Mineraldünger durch Vergasungsprozess anfallende Gärreste die Emissionsbilanz verbessern. Seit Ende 2006 die ersten Anlagen zur Einspeisung von Biomethan ins Erdgasnetz in Betrieb genommen wurden, ist eine zunehmende Professionalisierung und Industrialisierung der Biomethanerzeugung festzustellen. In diesem Zusammenhang ist in allen Prozessschritten mit einer starken Verbesserung zu rechnen, da bereits heute die marktreife, Optimierte Technik' günstigere Werte als bestehende Anlagen aufweist und weitere Verbesserungen erwartbar sind. 
- Dabei ist der derzeitige Bestand an noch überwiegend landwirtschaftlich betriebenen Biogasanlagen, die Biogas direkt zur Verstromung bzw. zur gekoppelten Strom- und Wärmeerzeugung bereitstellen, nicht berücksichtigt. Bei diesen können über die gesamte Prozesskette teilweise noch deutlich höhere Emissionen auftreten, die vor allem auf Leckagen von Methan (z.B. in nicht abgedeckten Gärrestlagern) zurückzuführen sind. Wird Wirtschaftsdünger als Substrat eingesetzt, kann allerdings vielfach eine Gutschrift für vermiedenen Emissionen der Lagerung angerechnet werden. Diese Biogasanlagen sind aber in keinem Fall mit der hier beschriebenen Biomethanerzeugung zu verwechseln.

- Insgesamt liegen die THG-Emissionen der Vorkette von - industriell bereitgestelltem - Biomethan bei derzeit rund 27,8 t CO 2 äq./TJ im Vergleich zu heutigen Emissionsfaktoren von 14,7 t $\mathrm{CO}_{2}$ - $\mathrm{Ä}$./TJ für die Vorkette russischen Erdgases oder 2,7 t $\mathrm{CO}_{2}$ - $\mathrm{Ä}$./TJ für Erdgas aus Deutschland. Bis 2030 erwarten wir eine Verringerung der spezifischen, d.h. auf den Energiehalt des eingespeisten Gases bezogenen, Vorketten-Emissionen von Biomethan um ca. die Hälfte auf 14,8 t $\mathrm{CO}_{2}$ - $\mathrm{Ä} . / \mathrm{TJ}$.

- Hinzu kommt der große Vorteil des biogenen Energieträgers: während das fossile Erdgas mit jedem verbrannten TJ Emissionen von $56 \mathrm{t} \mathrm{CO}_{2}$ produziert, entstehen bei der Nutzung von Biomethan fast keine klimarelevanten THGEmissionen. In der Summe weist die Nutzung von Biomethan unter Berücksichtigung der gesamten Prozesskette bereits heute um ca. 50 bis $60 \%$ geringere Gesamtemissionen auf als die Nutzung fossilen Erdgases. Dieser Vorteil kann bis 2030 auf $75 \%$ und mehr ansteigen.

Insgesamt zeigt sich, dass die Treibhausgasemissionen die mit der Nutzung gasförmiger Energieträger verbunden sind, vor allem von der Einsatzmenge abhängen. Das heißt, dass sowohl aus klima- als auch aus energiepolitischer Sicht die Steigerung der Energieeffizienz ein zentraler Faktor ist. Verstärkt werden kann dies aber durch eine Verbesserung der Klimaqualität, also der spezifischen mit der Nutzung einer Einheit des Energieträgers Gas verbundenen Treibhausgasemissionen. Denn sowohl in der verstärkten Nutzung von Biomethan als auch in der weiteren Investition in emissionsoptimierte Technologien entlang der Vorketten bestehen zusätzliche signifikante Emissionsminderungspotentiale. Die Vorteile gasförmiger Energieträger in der Klimaqualität und in der effizienteren Nutzung werden - bei entsprechender Modernisierung der Infrastrukturen - , verglichen mit anderen fossilen Energieträgern, auch zukünftig Bestand haben.

\section{Danksagung}

Die hier vorgestellten Ergebnisse wurden u.a. im Rahmen eines von E.ON Ruhrgas finanzierten Forschungsvorhabens erarbeitet. Wir danken unseren Institutskollegen Ole Soukup und Jochen Luhmann, den Kollegen und Kolleginnen des ifeu, DBFZ und TLL als Teilnehmern und Teilnehmerinnen des LCA Workshops Biogas sowie Frau Petra Nitschke-Kowsky und Herrn Alexander Vogel von E.ON Ruhrgas für ihre Unterstützung.

\section{Literatur}

Arnold, Karin (2010): Life cycle assessment of GHG mitigation of pro- duction and use of biomethane: sensitivity of effects from N2O emissions. J Integr Environ Sci, in Vorbereitung, 2010

Arnold, Karin \& Vetter, Armin (2010): Klima- und Umwelteffekte von Biomethan: Substrate und Anlagentechnik. Wuppertal Paper, Nr.182 BMU (2004). Ökologisch Optimierter Ausbau Erneuerbarer Energien. Bundesministerium für Umwelt, Naturschutz und Reaktorsicherheit, Berlin Bayerische Landesanstalt für Landwirtschaft LFL (2007): Klimabilanz für Biogasstrom. Klimabilanz der energetischen Nutzung von Biogas aus Wirtschaftsdüngern und nachwachsenden Rohstoffen. Freisingen.

BMWI (2006): BGB1. I S 2477; http:/www.bmwi.de/BMWi/Redaktion/PDF/E/entwurf-verordnungbiogas,property $=$ pdf, bereich $=$ bmwi, sprache $=$ de, $r w b=\operatorname{true} . p d f(24.02 .2009)$

CIEP (2004): Natural gas supply for the EU in the short to medium term. Clingendael International Energy Programme. The Clingendael Institute. Den Haag.

EnviTec (2007): Pressemitteilung bei der 6. Biogasfachtagung der EnviTec Biogas AG. Lohne/Saerbeck

European Parliament (2008): P6_TA-PROV(2008)0613 Monitoring and reduction of greenhouse gas emissions from fuels (road transport and inland waterway vessels) (Provisional edition - TEXTS ADOPTED at the sitting of Wednesday 17 December 2008);

http://www.europarl.europa.eu/sides/getDoc.do? pubRef $=-/ / E P / / T E X T+T A+20081217+I T E M S+D O C+X M L+V 0 / / E N \& l a n g u a g e=E N \#$ sdocta5. Abgerufen am 20. Juli 2009

Ewi (2005). Energie Report IV. Entwicklung der Energiemärkte bis zum Jahr 2030. Energiewirtschaftliches Institut an der Universität zu Köln

(Hrsg.). Oldenbourg Industrieverlag, München

Fachagentur Nachwachsende Rohstoffe e.V. (2006): Handreichung Biogasgewinnung und -nutzung. FNR, Gülzow.

Fachagentur Nachwachsende Rohstoffe e.V. (2008): Biogasbasisdaten Deutschland. FNR, Gülzow

Fachverband Biogas e.V (2007): Biogas im Jahr 2020: Wo werden wir stehen? Vortrag bei der 16. Jahrestagung des Fachverbandes Biogas. Leipzig.

Fraunhofer UMSICHT (2008): Technologien und Kosten der Biogasaufbereitung und Einspeisung in das Erdgasnetz. Ergebnisse der Markterhebung 2007-2008. Oberhausen.

Global Insight (2005): European Natural Gas Supply and Demand Report.

Gutser, R. (2008): Nährstoff- und Humuswirkungen bestimmen die Vorzüglichkeit organischer Dünger. Vortrag bei 5. Pflanzenbautagung vom 25.01.2008, TU München.

IEA (2004): World Energy Outlook 2004. International Energy Agency. Paris.

IEA (2005): Natural Gas Information 2005. International Energy Agency. Paris.

IEA (2006a): Natural Gas Market Review 2006. Towards A Global Gas Market. International Energy Agency. Paris.

IEA (2006b): Optimising Russian Natural Gas. Reform and Climate Policy. Paris.

IGU (2006): Report of Programme Committee B. Trennium 2003-2006. Strategy, Economy and Regulation. International Gas Union. Amsterdam. Institut für Energie- und Umweltforschung (2008): Basisdaten zu THG Bilanzen für Biogas-Prozessketten und Erstellung neuer THG-Bilanzen. Im Auftrag der E.ON Ruhrgas AG. Heidelberg. 
Institut für Energetik und Umwelt Leipzig, Thüringische Landesanstalt für Landwirtschaft, Fichtner GmbH; S. Klinski (2007): Monitoring zur Wirkung des novellierten Erneuerbare-Energien-Gesetzes (EEG) auf die Entwicklung der Stromerzeugung aus Biomasse. Im Auftrag des BMU. Leipzig, Stuttgart, Jena, Berlin.

IPCC (1995): IPCC/Second Assessment. Report (SAR). 'Climate Change 1995'. Cambridge University Press, UK

IPCC (2001): IPCC Third Assessment Report (TAR) 'Climate Change 2001'. Cambridge University Press, UK

IPCC (2007): IPCC Fourth Assessment Report: 'Climate Change 2007'. Cambridge University Press, UK

JRC (2003): WELL-to-TANK Report; Well-to-Wheels analysis of future automotive fuels and power trains in the European context. European Commission Joint Research Centre, Ispra

Kuratorium für Technik und Bauwesen in der Landwirtschaft e.V. (2007): Faustzahlen Biogas. KTBL, Darmstadt.

Landesumweltamt Brandenburg (2008): Überwachung von Biogasanlagen. Vortrag bei Fachtagung Biogas. Brandenburg, 23. Oktober 2008

Lechtenböhmer S \& Dienst C (2008): Treibhausgas-Emissionen zukünftiger Erdgas-Bereitstellung für Deutschland. UWSF - Z Umweltchem Ökotox 20 (2) 133-144

Lechtenböhmer, Stefan; Dienst, Carmen; Fischedick, Manfred; Hanke, Thomas; Fernandez, Roger: Tapping the leakages: methane losses, mitigation options and policy issues for Russian long distance gas transmission pipelines. In: International journal of greenhouse gas control, 1 (2007), 4, S. 387-395.

Luhmann, H.J., Lechtenböhmer, S., Venjakob, J. (2008): Energieträgerqualitätsziele - ein innovativer Ansatz zur Weitergabe der Synergie von Energieeffizienz- und REN-Verpflichtungen, Energiewirtschaftliche Tagesfragen 58. Jg. H. 6, S. 68-72

Medvedev (2006): Expanding Russian Gas Exports to the EU Gas Market undergoing Liberalization. Vortrag 23. Weltgaskonferenz, Amsterdam 2006.

Öko Institut (2006): Stand und Entwicklung von Treibhausgasemissionen in den Vorketten für Erdöl und Erdgas. Kurzstudie für das Institut für wirtschaftliche Oelheizung e.V. (IWO), Darmstadt

Soukup, Ole (2008): Erstellung von Produktökobilanzen auf Basis von Stoffstromnetzen für die Bereitstellung von Biogas zur Einspeisung in das Erdgasnetz. Diplomarbeit. Leuphana Universität, Lüneburg

Stern, J.P. (2006): The changing face of Gazprom. In: Fundamentals of the World Gas Industry 2006. Petroleum Economist, London, S. 35-39.

SWP (2006): Russlands Öl und Europa. Analyse von Götz, Roland (SWP) im Auftrag der Friedrich-Ebert-Stiftung, Berlin

Umweltbundesamt (2009) Nationaler Inventarbericht zum Deutschen Treibhausgasinventar 1990-2007. Berichterstattung unter der Klimarahmenkonvention der Vereinten Nationen. Dessau

Wuppertal Institut (2005) Treibhausgasemissionen des russischen Erdgas-Exportpipeline-Systems. Ergebnisse und Hochrechnungen empirischer Untersuchungen in Russland. Studie im Auftrag der E.ON-Ruhrgas AG, in Zusammenarbeit mit dem Max-Planck- Institut für Chemie, Mainz, Wuppertal

Wuppertal Institut, Institut für Energetik und Umwelt Leipzig, Fraunhofer UMSICHT, Gas-Wärme-Institut Essen (2006): Analyse und Bewertung der Nutzungsmöglichkeiten von Biomasse. Im Auftrag des BGW/ DVGW. Wuppertal, Leipzig, Oberhausen, Essen

www.biogas-netzeinspeisung.at/technische-planung/biogasgestehung/gasertraege1.html. Abgerufen am 17. Juni 2009

www.biogaspartner.de/index.php?id=11070\&L=axdixyfwre. Abgerufen am 1. Februar 2010 